\title{
CCB is Involved in Actin-Based Axonal Transport of Selected Synaptic Proteins
}

\author{
(D)Alfonso Martin-Peña ${ }^{1,2,3}$ and Alberto Ferrus ${ }^{1}$ \\ ${ }^{1}$ Department of Molecular, Cellular and Developmental Neurobiology, Cajal Institute, Madrid 28002, Spain, ${ }^{2}$ Department of Neurology, and ${ }^{3}$ Center for \\ Smell and Taste, McKnight Brain Institute, University of Florida, Gainesville, Florida 32611
}

\begin{abstract}
Synapse formation, maturation, and turnover require a finely regulated transport system that delivers selected cargos to specific synapses. However, the supporting mechanisms of this process are not fully understood. The present study unravels a new molecular system for vesicle-based axonal transport of proteins in male and female flies (Drosophila melanogaster). Here, we identify the gene CG14579 as the transcription unit corresponding to the regulatory mutations known as central complex broad ( $c c b$ ). These mutations were previously isolated for their morphological phenotype in R-neurons of the ellipsoid body, a component of the central complex. Mutant axons from R-neurons fail to cross the midline, which is indicative of an aberrant composition of the growth cone. However, the molecular mechanism remained to be deciphered. In this manuscript, we show that CCB is involved in axonal trafficking of FasII and synaptobrevin, but not syntaxin. These results suggest that axonal transport of certain proteins is required for the correct pathfinding of R-neurons. We further investigated the molecular network supporting the CCB system and found that CCB colocalizes and coimmunoprecipitates with Rab11. Epistasis studies indicated that Rab11 is positioned downstream of CCB within this axonal transport system. Interestingly, $c c b$ also interacts with actin and the actin nucleator spire. The data revealed that this interaction plays a key role in the development of axonal connections within the ellipsoid body. We propose that the CCB/Rab11/SPIRE system regulates axonal trafficking of synaptic proteins required for proper connectivity and synaptic function.
\end{abstract}

Key words: actin cytoskeleton; axonal transport; Drosophila; ellipsoid body; Rab11; synapses

\section{Significance Statement}

Proper function of the nervous system requires the establishment of mature, functional synapses. Differential protein composition in the synapse enables optimal performance of cognitive tasks. Therefore, it is critical to have a finely regulated transport system to deliver selected synaptic proteins to synapses. Remarkably, impairments in cytoskeleton-based protein-transport systems often underlie cognitive deficits, such as those associated with aging and neurodegenerative diseases. This study reveals that CCB is part of a novel transport system that delivers certain synaptic proteins via the actin cytoskeleton within the Rab11related domain of slow recycling endosomes.

\section{Introduction}

Axonal growth toward precise synaptic targets and delivery of synaptic components required for genesis and maturation of synapses are essential for proper function of the nervous system.

\footnotetext{
Received April 15, 2018; revised 0ct. 23, 2019; accepted 0ct. 24, 2019

Author contributions: A.M.-P. and A.F. designed research; A.M.-P. performed research; A.M.-P. contributed unpublished reagents/analytic tools; A.M.-P. and A.F. analyzed data; A.M.-P. wrote the paper.

This work was supported by Grants BFU2012-3819 and BFU2015-65685 from the Spanish Ministry of Economy to A.F., and by the Spanish Ministry of Education Fellowship BES-2004-3734 to A.M.-P. We thank the Bloomington Drosophila Stock Center (NIH P400D018537) and the Vienna Drosophila Resource Center for fly strains, and Amy S. Murphy for critical comments and proofreading of the paper.

The authors declare no competing financial interests.

Correspondence should be addressed to Alberto Ferrus at aferrus@cajal.csic.es or Alfonso Martin-Peña at alfonso.m.pena@ufl.edu.

https://doi.org/10.1523/JNEUROSCI.0915-18.2019

Copyright $\odot 2020$ the authors
}

Furthermore, these processes demand regulated protein trafficking across various intracellular compartments to ensure delivery to the correct synaptic destination. We set to investigate the molecular underpinnings of mutants known for their impaired locomotion and defective axonal pattern (Renn et al., 1999).

The Drosophila central complex broad ( $c c b$ ) mutants were initially identified for their locomotion phenotype during vertical upward walking (negative geotaxia), walking toward light sources (fast phototaxia), and walking toward landmarks on the Buridan's arena (Strauss and Heisenberg, 1993). The degree of these locomotor phenotypes varies considerably and correlates with anatomical defects in the central complex, which appears generally fragmented with the fan-shaped-body and the ellipsoid body (EB) affected by a lateral elongation (Strauss and Heisenberg, 1993). This phenotype is caused by the failure of R-neuron 
A

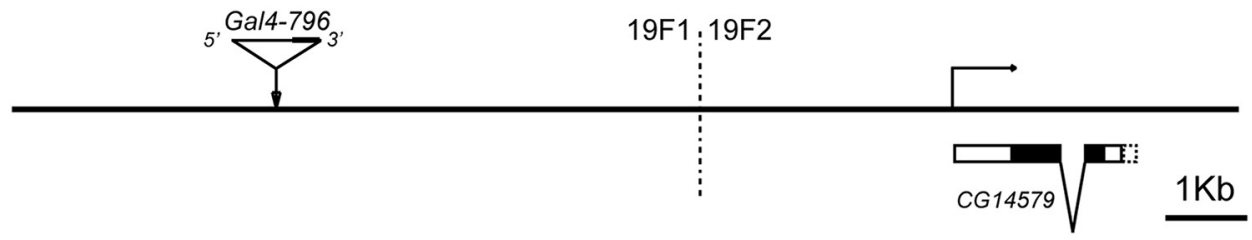

B

atgtcggcatcatttCGGTTAGATTTCGGTTCATTTTATCGAATTTCATTAGCGGCAACAGCGATTCCAATTTCCAATTTACTATTTCCATTTAGTCTGGGACTGGC ATTTTACAATTTTGCACTTAAACGCCCCCACCAGCCAATTGTTTCCTCCTCTGGCAATCCGGCTTCGAATGGGATTTTATTTATTTATTACGCACACGCAG CAAGTCCAAACACAGCGCATTCCTTTGTCCTCGTGGGTCCTTTGTCCTTTCAGACGAACTGAGCAAATATTTAGAAATATTTGGGCGTCGAAAATCGGAC TCGTCTCCCCAGGGCCATGAATAAATTAATTGACCAGCCCACAAGTATTTAAGCTTGGTCACGAAAACATCATAGTTTTAGTTATCGAAAGTCGCGAGCA CAATAGCGAACATAGGATTTTACTATTTATAATCAAAGCGGTGTATCTTTCTTTTCCGCTTACCTATTTAAGATTCTAAAATGTTGTGTAAAGTATTGGTAG TACATTGAAACCTTTTTGAATAGGCCTGGTGAAAGTACTCAGTACAATGTCATTTAGGCTCTATGACAGATTTGCCGTACCATTCAGTCTCAGCCAGAGCT CCGTTGGCTGAACAACAAAATCAAAATTTATATTTTTTTACAAGTTTTTCCATAAAAAGAAGTGCATCCGTTTTCTAAGCAAGAAAGACGAAAAATGAC CATAATGGCTATATCAAACGACACATTTGAAAATGAACAGCAGGTGGCGGTAAGGCAAGAAGCACCCAATGCCATTGGTAACATCCCGGATGTGG TGGAGTCGGCTTCCCGCCCACAAAAATACACGTTCCATGAGATCTATCAGATGATTACAGCCCTCACCTGGCCCCGAATTCTGTTTGATTTGCTCG TGCGCCACGAGTGGAACTCCATCTTTTTGAGCCTAGGCTTTCTTATCCTCAATGCCTTCGAGGTCCTTCAAAGCTTCAATCTGTTGCAACAGCTG GCTGTAGTCGGCATGATGATCCTGAGCAACATAATGATCTACAAGGCCATCCGGCCGTATGTGCGATTCCATCTTTACGAGCGTCTGATCCGCGC CGATTTCAATGTGCACATCACCCAGGAAATGGTTGTGCGCGTGGTCAACTGTGTAAATCGCTGCTTGGTCACGCTGCATTGCATCCTCTTTCAAA CTGAAATCTTCGAGCTCTTTTTGATGATGCTTGCCCTGGAGGCAGTGCGTAACATCATCTGGTATACTAACATCTTCTTGCTAATTAGGATCGgtaag ttttccatatgcgacctcaaatgaacacacgcaaattattccaatcaagcttttattgtttttagtattacttttaatatatattaagaaatgcaatetctettgcagTCTTATGTGTGACGTTGTTTTTTCCAAGGCTTTTC GATAATGATATTGATAATGCGTCGAGCAGAGCCTATTATCAGATATGTGTGCTGCAGATCGTTGACAAGCTTGAGGAGATAGGCGTGGAGGTGCA TCTAGGCATCATTGAGCGCTGGAAGCAGGAAGTGGATGCCATGAGGGAGTTGTATTACGAGATTGAGGACTGGCCAAACCAAGAGTAGTTTCATG ATGATATCGAGATCTAGTAAAATATATGGCTTCGCCGAGGATAATAGCCCATTATGGCAGTTATGGATAACTTTTGTATTTAACTTCTTAATTGTTTCCATA CACTCCATACAACTACAATGCCAAATTCGCACAATCTACCTATCAAAAACATCAAATTACACCTTACACTTTAAATTTGTCCTTGCTTTATTTCATTTAaatt $a a a$ TTATGTACACTGCACACTGAAAAACTGTCTCCTGTTTCATTTAATTTAAAGGTTAAAGGAAAGCCCATTGAGTACCAGGCTTATATTGAGGGAAATAA CCGGGTTGTGAAGTGGTCAAATCTGATTGATTTTCGGTGTGGAAATTaattaaagcc

\section{C}

MTIMAISNDTFENEQQVAVRQEAPNAIGNIPDVVESASRPQKYTFHEIYQMITALTW PRILFDLLVRH EWNSIFLSLGFLILNAFEVLQSFNLLQQLAVVGMMILSNIMIYKAIR PYVRFHLYERLIRADFNVHITQ EMVVRVVNCVNRCLVTLHCILFQTEIFELFLM MLALEAVRNIIWYTNIFLLIRI-VLCVTLFFPRLFDND IDNASSRAYYQICVLQIVDK LEEIGVEVHLGIIER W K QEVDAMRELYYEIEDWPNQE

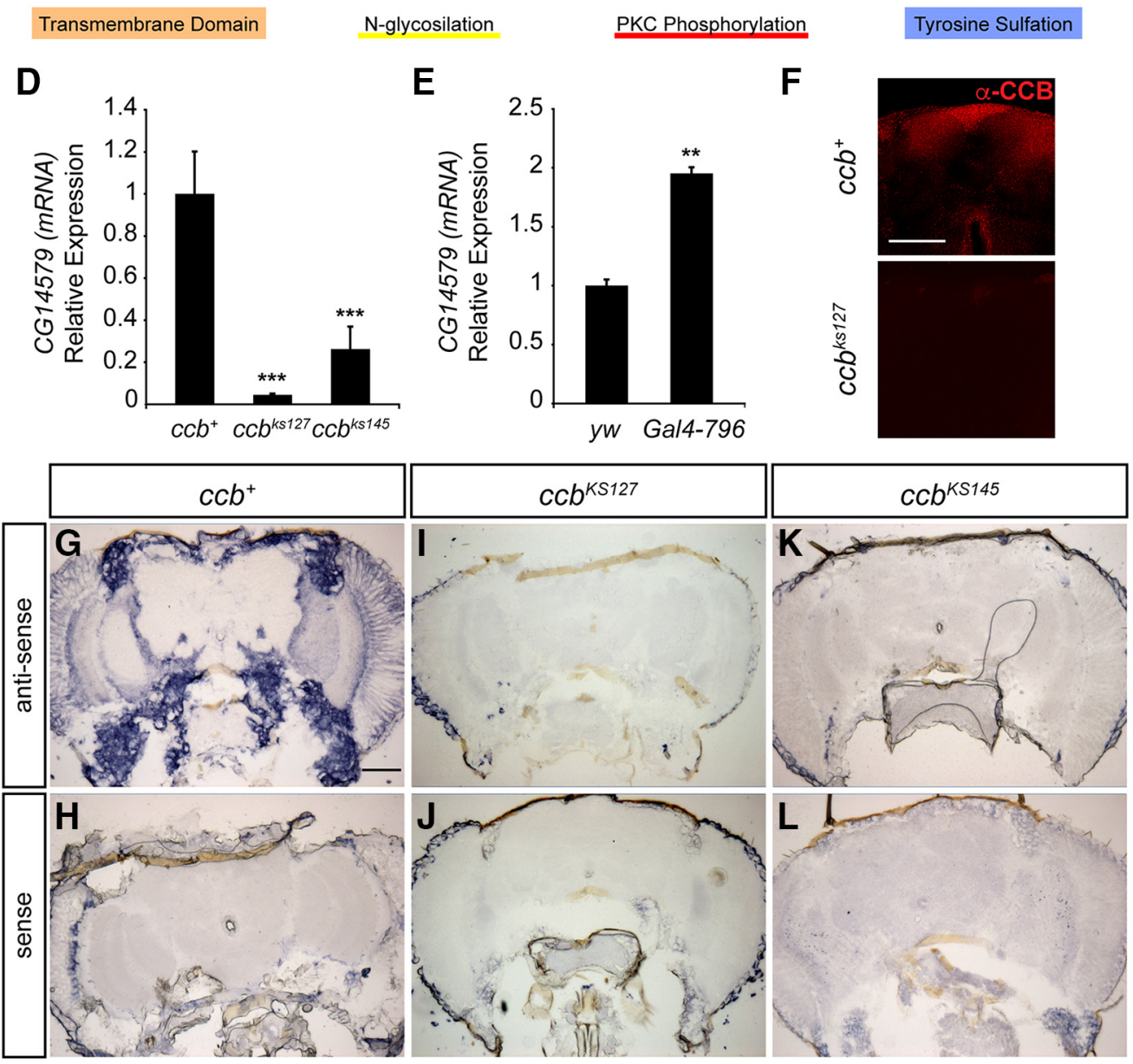

Figure 1. $c c b$ mutations affect (G14579 expression. $A$, Schematic representation of the genomic region in the boundaries between polytene chromosome bands $19 F 1$ and $19 F 2$ indicating the relative position of the insertion point for Gal4-796 and the (G14579 transcription unit. The orientation of the Gal4-796 element is indicated as well as the molecular organization of the transcription unit (G14579 into exons (black boxes), intron (V gap), and untranslated regions (UTRs; empty boxes). B. Nucleotide sequence of the transcription unit (Figure legend continues.) 
Table 1. Potential phosphorylation sites in CCB

\begin{tabular}{lcl}
\hline Amino acid & Position & $p$ \\
\hline Ser & 36 & 0.701 \\
Ser & 211 & 0.664 \\
Tyr & 49 & 0.812 \\
Tyr & 254 & 0.936 \\
\hline
\end{tabular}

Table 2. Predicted CCB-phosphorylating kinases

\begin{tabular}{lll}
\hline Kinase & Position, aa & $p$ \\
\hline PKA & $76 \mathrm{~S}$ & 0.75 \\
PKG & 44T & 0.66 \\
PKC & $56 \mathrm{~T} / 153 \mathrm{~T} / 2115$ & $0.88 / 0.52 / 0.75$ \\
CKII & $75 / 161 \mathrm{~T}$ & $0.52 / 0.52$ \\
ATM & $136 \mathrm{~T}$ & 0.51 \\
SRC & 254Y & 0.52 \\
CDC2 & $56 \mathrm{~T}$ & 0.52 \\
\hline
\end{tabular}

Table 3. Predicted transmembrane domains in CCB

\begin{tabular}{lllllll}
\hline Software & Domain 1 & Domain 2 & Loop & Domain 3 & Domain 4 & N-/C-terminal \\
\hline SMART & $70-89$ & $93-111$ & 40 & $151-173$ & $178-200$ & - \\
TMHMM & $70-89$ & $93-111$ & 40 & $151-173$ & $178-200$ & Internal \\
SOSUIsignal & $68-90$ & $94-116$ & 39 & $155-177$ & $181-203$ & - \\
PSORT & $72-88$ & $98-114$ & 43 & $157-173$ & $183-199$ & Internal \\
PSORT II & $72-88$ & $98-114$ & 43 & $157-173$ & $183-199$ & Internal \\
HMMTOP & $70-91$ & $97-118$ & 32 & $150-173$ & $179-199$ & External \\
HMMTOP II & $71-88$ & $97-114$ & 42 & $156-173$ & $180-197$ & External \\
DAS-Tmfilter & $73-85$ & $97-108$ & 53 & $161-174$ & $180-199$ & - \\
SVMTM & $73-87$ & $96-110$ & 46 & $156-174$ & $180-198$ & - \\
Impred & $70-88$ & $95-114$ & 70 & - & $184-200$ & Internal \\
\hline
\end{tabular}

axons to cross the midline, resulting in the formation of two independent EBs (Renn et al., 1999). However, the mechanism causing this morphological phenotype remains unknown. We reasoned that this phenotype could be related to defective axonal transport.

\footnotetext{
$\leftarrow$

(Figure legend continued.) (G14579. Genomic sequence is indicated in lower case while the nucleotides covering the transcribed messenger are in upper case. The transcription initiation site (italic bold) is located $8270 \mathrm{nt}$ downstream of the insertion point for Gal4-796. This transcription unit contains a $5^{\prime}$ UTR ( $698 \mathrm{nt}$ ), from the transcription initiation site (atcattt) to the translation initiation triplet, and two alternative 3' UTR (214 and 367 nt), from the stop codon to each of the two polyadenylation sequences (aattaaa). The two exons (bold, uppercase), of 574 and $218 \mathrm{nt}$ respectively, covering the ORF are separated by a single intron of $213 \mathrm{nt}$. C, Amino-acid sequence of the encoded protein with predicted domains and posttranslational sites highlighted: transmembrane domains (orange), Tyr sulfation (blue), N-glycosilation (yellow), and PKC phosphorylation (red). The monoclonal antibody anti-CCB was generated against a small peptide in the N-terminal region of the protein (bold underlined). $\boldsymbol{D}, \boldsymbol{E}$, Quantitative RT-PCR showing the relative expression levels of the (G14579 mRNA in heads from 3-5 days old adult males. D, Flies hemizygous for $c c b^{k s 127}$ or $c c b^{k 5145}$ mutations express the mRNA at a significantly lower level than the age-matched control flies (Kruskal-Wallis, $p<0.0001$ ). The data were normalized to the expression level in wild-type flies from the same genetic background, Berlin $\left(c c b^{+}\right)$. E, Flies carrying the insertion Gal4 -796 express the (G14579 mRNA at a significantly higher level than the corresponding control flies (Kruskal-Wallis, $p=0.0090$ ). These values were normalized to the expression level of the parental line $y$ w. $\boldsymbol{F}$, Adult brain of control ( $c c b^{+}$, top) and mutant ( $c c b^{k s 127}$, bottom) male flies stained against $C(B B$ validate the antibody specificity. Scale bar $30 \mu \mathrm{m}$. $\mathbf{G}-\boldsymbol{L}$, In situ hybridization for $(G 14579$ on cryosectioned heads from control $c c b^{+}$(wild-type Berlin; $\boldsymbol{G}, \boldsymbol{H}$ ) and mutant $c c b^{k s 127}(\boldsymbol{I}, \boldsymbol{J})$ and $c c b^{k s 145}(\boldsymbol{K}, \boldsymbol{L})$ adult male heads. Cryosections were incubated with the antisense probe corresponding to the C $G 14579$ messenger $(\boldsymbol{G}, \boldsymbol{I}, \boldsymbol{K})$ or the sense probe $(\boldsymbol{H}, \boldsymbol{J}, \boldsymbol{L})$ as negative control. The staining with the antisense probe reveals expression of the (G14579 mRNA all throughout the brain cortex in control flies $\mathrm{CCb}^{+/+}$(wild-type Berlin; $\boldsymbol{G}$ ), this expression is virtually absent in brains from $c c b^{k s 127 / k s 127}(\boldsymbol{I})$ and $c c b^{k s 145 / k s 145}(\boldsymbol{K})$ mutant flies. Error bars indicate SEM. ${ }^{* *} p<0.01,{ }^{* * *} p<$ $0.001 ; n=3$ per group. Scale bars: $\mathbf{G}-\boldsymbol{L}, 30 \mu \mathrm{m}$.
}

Two main transport systems are known based on the supporting cytoskeletal network: (1) a high-speed, long-range microtubulebased system; and (2) a slow-speed, local-range actin-based system. Microtubule-based transport uses motor proteins of the kinesin and dynein families binding to the plus (anterograde) and minus (retrograde) end of microtubules, respectively (Hirokawa et al., 2010; King, 2018). Myosin is the corresponding motor protein along actin filaments. Dynamic regulation of the actin filaments is involved in the initial steps of synaptogenesis (Chia et al., 2014) and neuritogenesis (Meyer and Smith, 2006).

Actin filaments are dynamic polymers that require the seeding effect of nucleation factors. Although Actin is ubiquitously present in all cell types, it can form mechanistically different types of filament networks according to the nucleator factor that triggered the network formation. The Arp $2 / 3$ complex, the Formin family and the Spire family are the three known families of Actin nucleator factors (Quinlan et al., 2005). Targeting specific nucleators to discrete cell compartments satisfies the local requirements of the cell (Rottner et al., 2017). Members of the Spire nucleator family have crucial roles in intracellular membrane organization and in the long range transport of vesicles and organelles (Tittel et al., 2015; Pylypenko et al., 2016).

Associated with the two supporting networks, Rab4 operates in the microtubule-based transport while Rab11 works in the actin-based transport (Grosshans et al., 2006; Pylypenko et al., 2016). Rab proteins are a class of Ras GTPases that regulate vesicle-based motor-protein-mediated transport, and each Rab member regulates a specific step of intracellular transport (Grosshans et al., 2006). Rab GTPases are molecular switches, swapping between GTP-bound active and GDP-bound inactive states to regulate transport. Rab proteins interact with regulatory proteins, such as guanine-exchange factors, GTPase-activating proteins, GDP-dissociation inhibitors, and GDP-displacement factors, which control the switch between the two states. Activated Rab proteins mediate their downstream effects via interaction with effector proteins that confer specificity of the compartment and the cargo protein. For instance, Rab11 couples to myosin in the recycling compartment to regulate the turnover of the transferrin receptor and several G-protein-coupled receptors (Sönnichsen et al., 2000), including receptors involved in axonal growth and guidance. This axonal growth and guidance requires intense and precisely timed transport of signaling molecules required in the surface of the growth cone.

To further understand the molecular mechanisms of protein trafficking, we investigated the role of $c c b$ in axonal transport. Here we show that $c c b$ regulates the traffic of selected proteins, including FasII and Synaptobrevin but not Syntaxin. Our results unraveled a vesicle-based protein trafficking system based on the Actin network and dependent on the Actin nucleator factor SPIRE. This system is positioned within the Rab11 domain of slow recycling endosomes ensuring successful delivery of synaptic proteins from the ER to synapses.

\section{Materials and Methods}

\section{Fly strains}

Fly stocks were raised on standard cornmeal food at $25^{\circ} \mathrm{C}$ and $70 \%$ relative humidity on a $12 \mathrm{~h}$ light/dark cycle. Flies carrying mutations in the $c c b$ gene $\left(c c b^{k s 127}\right.$ and $\left.c c b^{k s 145}\right)$ were provided by Roland Strauss, Center for Genetic and Neurobiology at Wurzburg University, Wurzburg, Germany (Heisenberg et al., 1985; Strauss and Heisenberg, 1993). These flies were backcrossed against wild-type Berlin for at least six generations before the experiments were performed. Flies carrying the mutation in the spire locus ( spir $^{1} \mathrm{cn} \mathrm{bw}$ ) were obtained from the Bloomington Stock Center (BDSC, catalog \#5113; RRID:BDSC_5113; http://flystocks. 

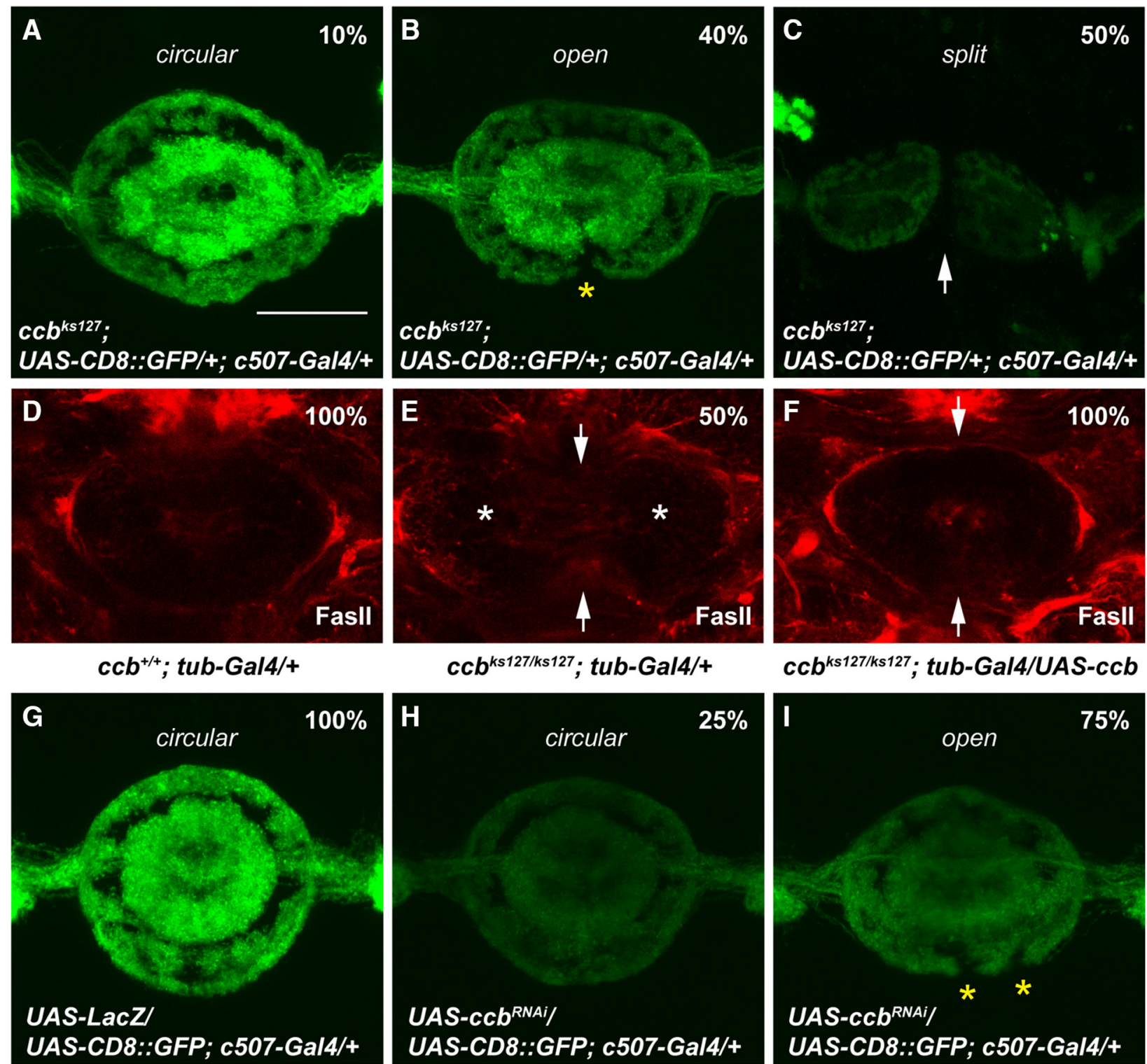

$c c b^{k s 127 / k s 127} ;$ tub-Gal4/+

$c c b^{k s 127 / k s 127} ;$ tub-Gal4/UAS-ccb
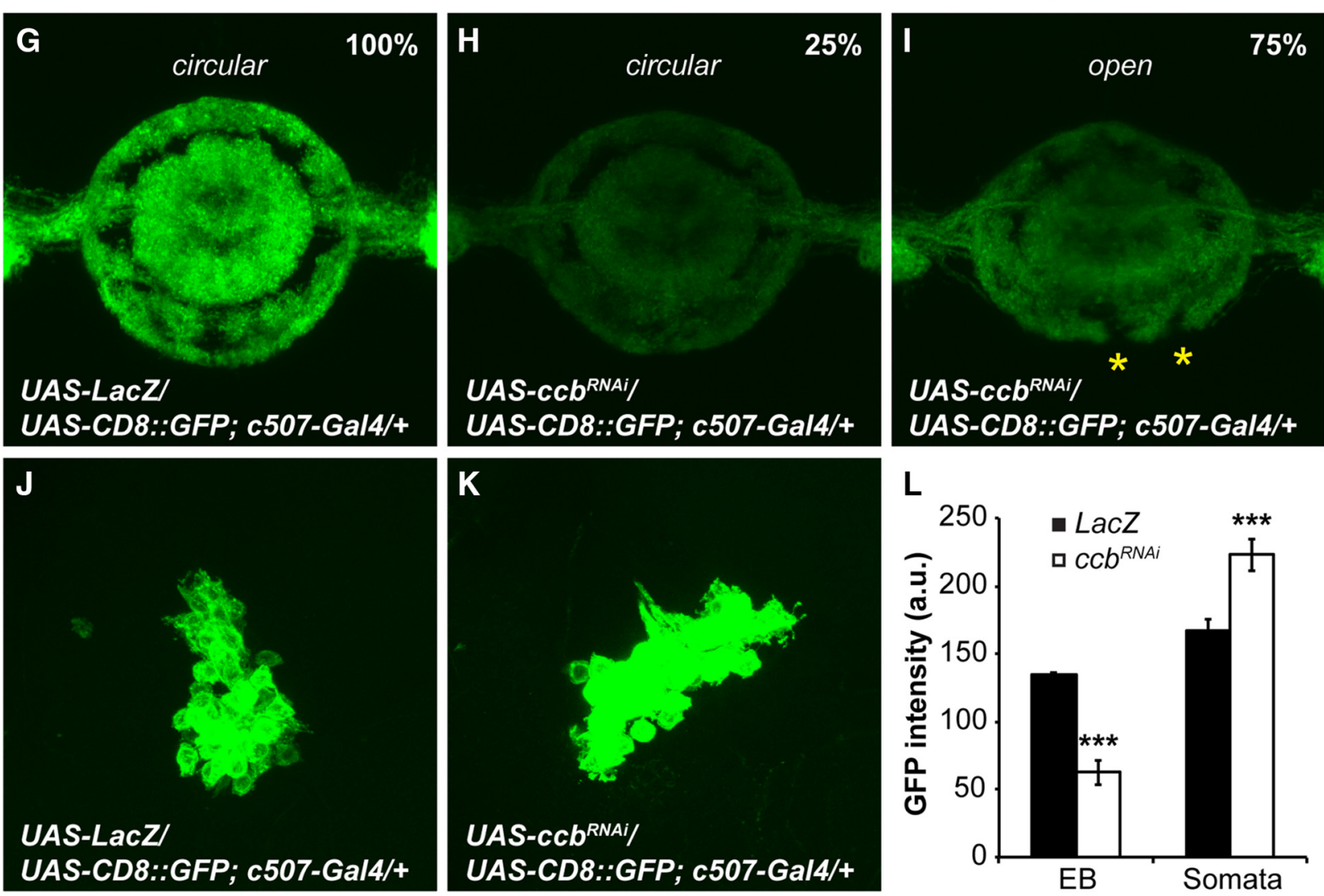

Figure 2. The expression level of $C G 14579$ dictates the penetrance of the $c c b$ phenotype in the EB. $A-C$, Morphology of the EB in adult males carrying the mutation $c c b^{k s 127}$ and expressing $C D 8$ :: GFP in R-neurons (cc ${ }^{k s 127}$; UAS-CD8::GFP/+; C507-Gal4/+). A, 10\% of these flies displayed an EB with a "circular" shape typical of wild-type flies. B, 40\% exhibited a ventrally "open" EB (various degrees). $\boldsymbol{C}, 50 \%$ presented a "split" EB. $\boldsymbol{D}-\boldsymbol{F}, \alpha$-Fasll staining of the EB in female control flies $\left(\boldsymbol{D} ; \boldsymbol{c c}^{+/+} ;\right.$tub-Gal/4/+), flies exhibiting the "split" phenotype with a $50 \%$ penetrance $(\boldsymbol{E}$; $c c b^{k S 127 / k s 127} ;$ tub-Gal4/+) and flies expressing the $c c b^{c D N A}\left(\boldsymbol{F} ; c c b^{k S 127 / k s 127} ;\right.$ tub-Gal4/UAS-cc $\left.{ }^{C D N A}\right)$, which rescues the developmental phenotype with a $100 \%$ penetrance. $\boldsymbol{G}, \boldsymbol{H}$, EB of flies expressing

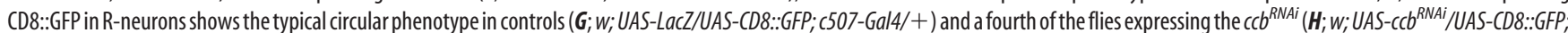

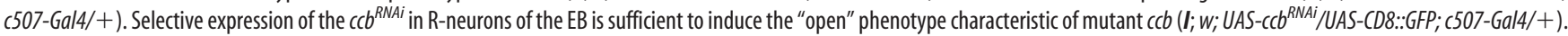
$\boldsymbol{J}, \boldsymbol{K}$, R-neurons somata of control $(\boldsymbol{J})$ and $c c b^{R N A i}$ expressing flies $(\boldsymbol{K})$. $\boldsymbol{L}$, Quantification of the GFP signal in the EB $(\boldsymbol{G}, \boldsymbol{H})$ and somata $(\boldsymbol{J}, \boldsymbol{K})$ of R-neurons. Expression of the $c c b^{R N A i}$ in R-neurons significantly reduces axonal transport of CD8::GFP to the EB (Mann-Whitney, $p<0.0001$ ) that it is accumulated in the soma (Figure legend continues.) 
bio.indiana.edu/). Driver lines 796-Gal4, c507-Gal4 (BDSC, catalog \#30840; RRID:BDSC_30840), c232-Gal4 (Martín-Peña et al., 2014), and D42-Gal4 (Martín-Peña et al., 2006) were previously described. Two independent overexpression lines for $c c b, U A S-c c b^{c D N A}$, were used; one was produced in our laboratory (UAS-cc $b^{c D N A}-a t t P 2$; Fig. 3-1, available at https://doi.org/10.1523/JNEUROSCI.0915-18.2019.f3-1) and the other one (UAS-ccb $b^{C 4}$-attP40) was obtained from the Exelixis Collection at the Harvard Medical School. Line UAS-cc $b^{R N A i}$ was generated in our laboratory (Fig. 3-1, available at https://doi.org/10.1523/JNEUROSCI. 0915-18.2019.f3-1) and UAS-spire ${ }^{R N A i}$ (VDRC 44092) was obtained from the Vienna Drosophila Resource Center (Dietzl et al., 2007). Reporter UAS lines UAS-Synb::GFP and UAS-CD8-GFP (BDSC, catalog \#5137; RRID:BDSC_5137) were previously reported (Martín-Peña et al., 2006). Lines UAS-Rab11::GFP (BDSC, catalog \#8506; RRID:BDSC_8506),

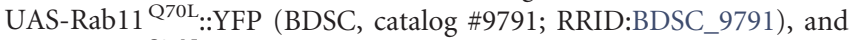

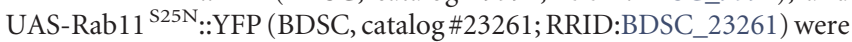
obtained from the Bloomington Stock Center.

Localization of the genomic insertion point of the pGawB-796 The localization of the insertion point for $\mathrm{p}(\mathrm{GawB})-796$ was performed by plasmid rescue (O'Kane and Gehring, 1987). Genomic DNA was obtained from adult flies $y w$ Gal4-796. One hundred flies were homogenized in extraction buffer ( $100 \mathrm{~mm}$ Tris- $\mathrm{HCl}, \mathrm{pH} 8,80$ mM EDTA, 100 $\mathrm{mm}$ glucose). Homogenates were incubated in $0.5 \%$ SDS and $0.3 \mu \mathrm{g} / \mu \mathrm{l}$ proteinase $\mathrm{K}$ at $65^{\circ} \mathrm{C}$ for $1 \mathrm{~h}$. Then, samples were treated with RNase followed by purification/precipitation and resuspended in TE buffer $(0.5$ м Tris- $\mathrm{HCl}, \mathrm{pH} 8,0.5$ м EDTA, pH 8). $3 \mathrm{mg}$ of genomic DNA were digested with PstI restriction enzyme to then self-ligate and be transformed into Max-DH10B (ThermoFisher Scientific, catalog \#18297010). Genomic fragments were enzymatically digested and sequenced to obtain the precise insertion point.

\section{Generation of UAS constructs and transgenic lines}

The open reading frame (ORF) of CG14579, the putative $c c b$ gene, was amplified by RT-PCR from Canton-S wild-type mRNA using the forward primer $5^{\prime}$-CCATATATCAGCGGCCGCATTTGAAAATGAACA GCAGGTG-3' and the reverse primer 5'-GCGGTACCGGTTTGGCC AGTCCTCA-3' and cloned into Kpn-I and Not-I restriction sites of pBlueScript KSII+ (Stratagene, catalog \#212207).

P-element $U A S$ - $c c b^{R N A i}$. We used a genomic-cDNA hybrid construct for RNA interference; this design has been proven to efficiently silence target genes in Drosophila (Kalidas and Smith, 2002; Romero-Pozuelo et al., 2007). The ORF was previously cloned into pBlueScript KSII+ (see previous section), and the genomic fragment was amplified from Canton-S genomic DNA using the same primers and cloned into Kpn-I [New England BioLabs (NEB), catalog \#R0142] and Not-I (NEB, catalog \#R0189) restriction sites of pBluescript. Both fragments were digested with Pst-I (NEB, catalog \#R0140) restriction enzyme cutting the second exon. The cDNA fragment was introduced in a reverse orientation at this Pst-I restriction site of the second exon in the genomic fragment. This yielded a pBluescript construct containing a 778bp genomic-665bp cDNA (reverse), which was transferred from pBluescript to the pUASt vector (https://www.addgene.org/vector-database/4473/) by digesting with the Kpn-I restriction site. Directionality of this insertion was checked by enzymatic restriction pattern and sequencing.

P-element UAS-ccb $b^{c D N A}$. The CG14579 cDNA was subcloned into Xho-I (NEB, catalog \#R0146) and Xba-I (NEB, catalog \#R0145) restriction sites of pUASt-attB (https://www.addgene.org/vector-database/ $5556 /$ ) and later inserted in the chromosomal band 22A (2L) for Gal4driven overexpression in different populations of somatic cells.

$\leftarrow$

(Figure legend continued.) (Mann-Whitney, $p<0.0001)$. Percentage in the top right corner indicates penetrance of the phenotype. White asterisks point the two EBs formed on each side of the midline (arrows). Yellow asterisks denote axonal growth deficits where axon terminals from R-neurons did not complete the ring and led to an open phenotype, characteristic of $c c b$ mutants. Scale bars: $\boldsymbol{A}, \boldsymbol{B}, 30 \mu \mathrm{m} ; \boldsymbol{C}, 60 \mu \mathrm{m} ; \boldsymbol{D}-\boldsymbol{K}, 30 \mu \mathrm{m}$. Error bars indicate SEM. ${ }^{* *} p<0.001$; $n=8-12$ per group.

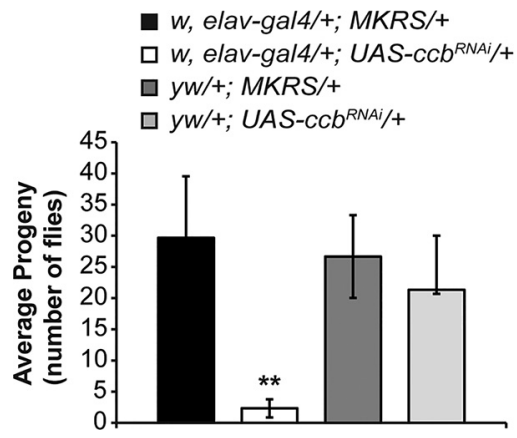

Figure 3. Pan-neuronal expression of the $c c b^{R N A i}$ induces lethality. Average number of flies in the progeny obtained from crossing UAS-cc $b^{R N A i}$ females with males of the pan-neuronal driver elav-Gal4. Pan-neuronal knockdown of $c c b$ induces lethality during development with only occasional escapers. Error bars indicate SEM. ${ }^{* *} p<0.01 ; n=10$. See Figure $3-1$, available at https://doi.org/10.1523/JNEUROSCI.0915-18.2019.f3-1.

\section{Quantitative RT-PCR. mRNA extraction}

Approximately 100 flies (100 mg) of each genotype were collected and processed with the QuickPrep micro mRNA Purification Kit (GE Healthcare Life Sciences, catalog \#27-925501) following the manufacturer's instructions. mRNA was quantified and precipitated to obtain a final working concentration of $150 \mathrm{ng} / \mu \mathrm{l}$.

Reverse-transcription reaction. mRNA (150 ng) and Oligo-dT $(0.2 \mu \mathrm{g})$ were used in each reaction of reverse transcription to cDNA using the First-Strand cDNA Synthesis Kit (GE Healthcare Life Sciences, catalog \#27-926101) according the manufacturer's instructions.

PCR. Reverse-transcription products were used as templates for PCRs by using several dilutions to generate the corresponding standard curves. We used the $140 \mathrm{kDa}$ RNApol-II subunit encoding-gene as normalizing internal control. Specific primers for the RNApolII and $c c b$ genes were designedfrom databanksequences. Forward primer:5'-GCAGTGCGTAA CATCATCTGGTATA-3' and reverse primer: 5' -CGAAAAGCCTTGGA AAAAACAACG-3' were used to amplify a $88 \mathrm{bp}$ fragment of the $c c b$ mRNA, FAM-dye-labeled TaqMan probe was: 5'-TCACACATAA GACGATCC-3'. Forward primer: 5'-ACTGAAATCATGATGTACGA CAACGA- $3^{\prime}$ and reverse primer: 5'-TGAGAGATCTCCTCGGCATT CT-3' were used to amplify a 68 bp fragment of the RNApolII mRNA, FAM-dye-labeled TaqMan probe was: 5' -TCCTCGTACAGTTCTTCC$3^{\prime}$. Primers and probes were added to TaqMan Universal PCR Master Mix (ThermoFisher Scientific, catalog \#4304437). The amplification was performed in an ABI PRISM 7000 sequence detector using the following conditions: $2 \mathrm{~min}$ at $50^{\circ} \mathrm{C}, 10 \mathrm{~min}$ at $95^{\circ} \mathrm{C}, 40$ cycles $\left(15 \mathrm{~s}\right.$ at $95^{\circ} \mathrm{C}, 60 \mathrm{~s}$ at $60^{\circ} \mathrm{C}$ ), and using the dissociation curve protocol. All amplified products were verified by sequencing. To calculate the relative index of gene expression, we used the efficiency-calibrated mathematical method. It is based on the algorithm Ratio $=\left[(E \text { target })^{\mathrm{Ct} \text { sample }} /(E \text { ref })^{\mathrm{Ct} \text { sample }}\right] /$ $\left[(E \text { target })^{\mathrm{Ct}}\right.$ calibrator $\left./(E \text { ref })^{\mathrm{Ct} \text { calibrator }}\right]$, where $(E$ ref $)$ is the reaction efficiency for the RNApolII ( $E$ target) corresponds to the $c c b$ gene, $\mathrm{Ct}$ sample is the average threshold cycle of the gene from the sample of the experimental genotype, whereas Ct calibrator corresponds to the average cycle of the gene from the sample of the control group. The $E$ values of each reaction are calculated from the slope of the corresponding standard curve according to $E=10^{-1 / \text { slope }}$.

\section{In situ hybridization}

Heads from young adults were cryosectioned and fixed in $4 \%$ paraformaldehyde at $4^{\circ} \mathrm{C}$ for $10 \mathrm{~min}$ (Winther et al., 2003). Sections were prehybridized, and then hybridized with antisense or sense $c c b$-specific probes (1:100) in hybridization solution (Winther et al., 2003). Linearized pBlueScript vector with the 792-bp cDNA fragment of $c c b$ (as described) was used as template for Digoxigenin-RNA labeling according to manufacturer's instructions (Boehringer). T3- and T7-RNA polymerase promotors flanking the cloning site of $c c b$ in pBlueScript were used for transcription of $c c b$ in the presence of DIG-UTP. Probe binding was detected in the samples by alkaline phosphatase-conjugated antiDigoxigenin (1:2000) for $1 \mathrm{~h}$. Detection of alkaline phosphatase was 


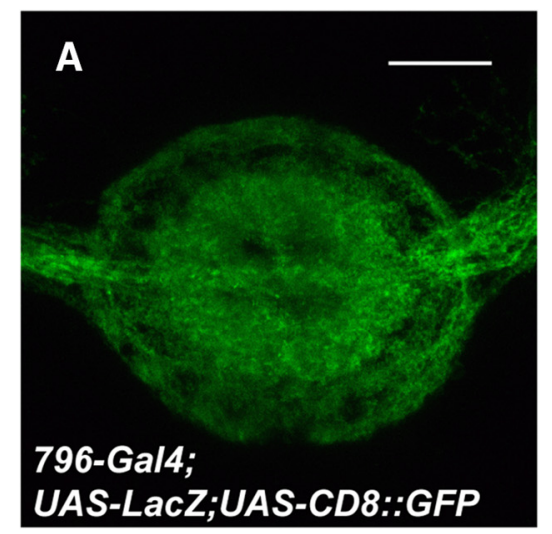

D

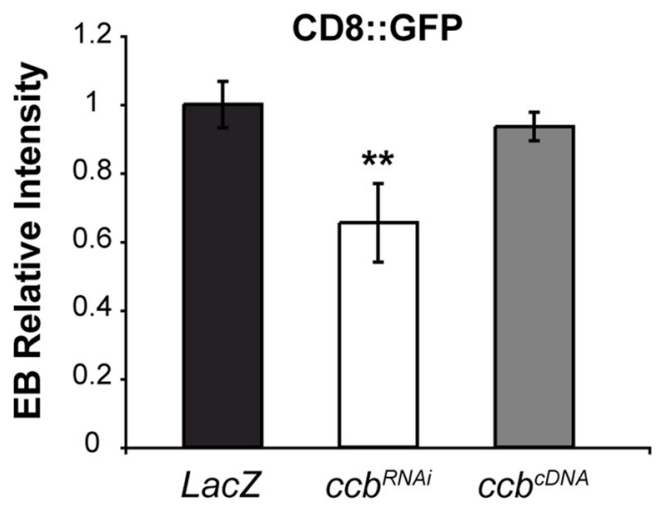

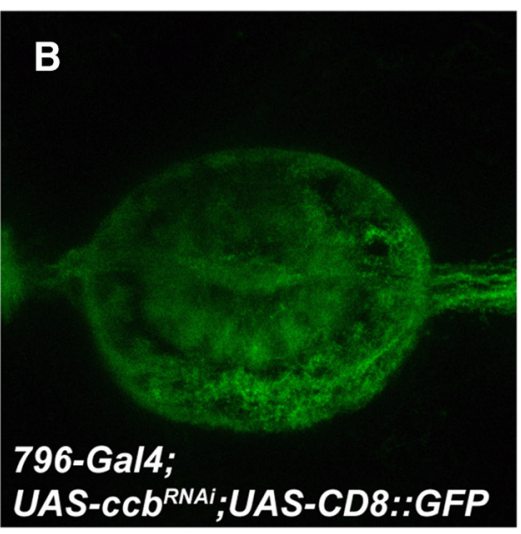

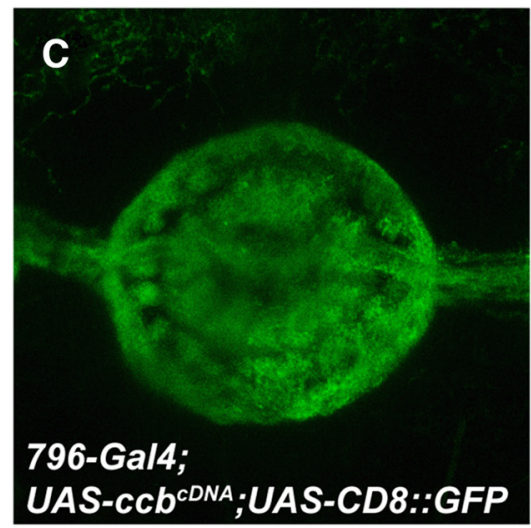

E

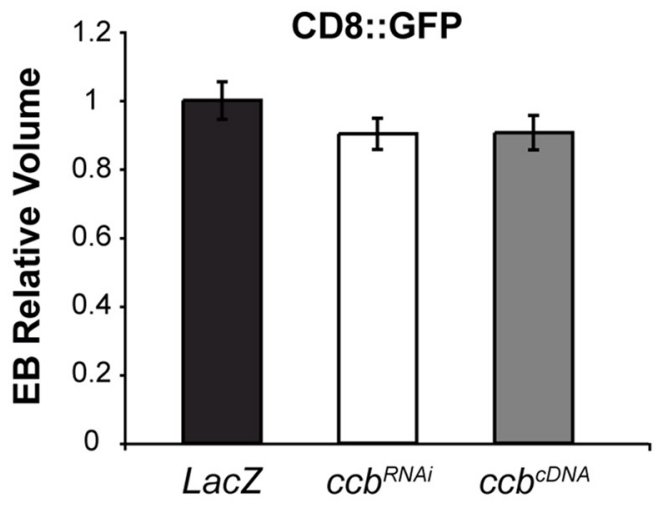

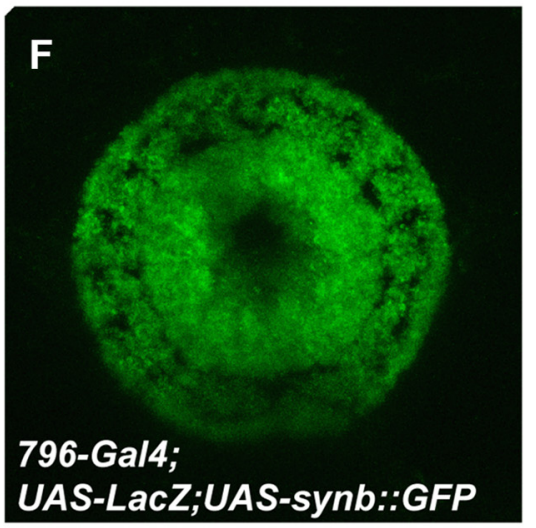

I

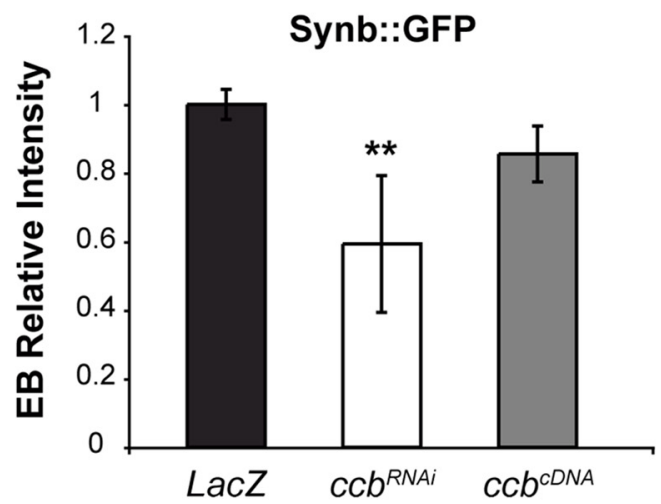

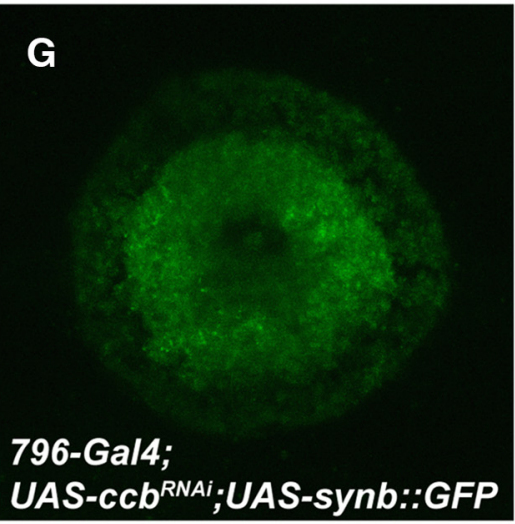

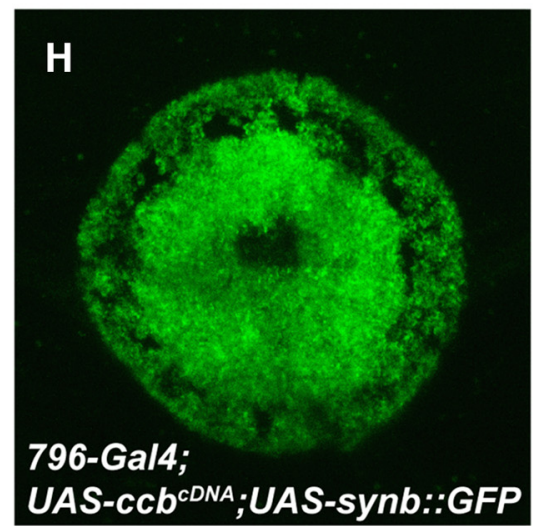

J

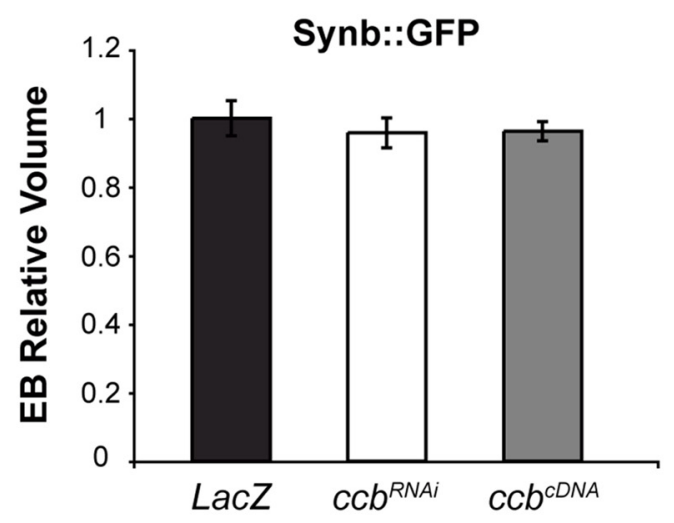

Figure 4. Axonal transport in adult R-neurons of the EB. Quantification of axonal transport of CD8::GFP $(\boldsymbol{A}-\boldsymbol{E})$ or Synaptobrevin::GFP $(\boldsymbol{F}-\boldsymbol{J})$ in R-neurons of the EB with downregulated or upregulated $c c b$. D, $\boldsymbol{I}$, Axonal transport of CD8 and Synaptobrevin in R-neurons is shown as the average GFP intensity of CD8::GFP (D) and Synaptobrevin::GFP $(\boldsymbol{I})$ in the EB and normalized to the GFP intensity in controls. Expression of the $c\left(b^{R N A i}\right.$ in R-neurons significantly reduces axonal transport of CD8 ( $\boldsymbol{B}$; Kruskal-Wallis, $p=0.0072$ ) and Synaptobrevin (Figure legend continues.) 
made in nitroblue tetrazolium/5-bromo-4-chloro-3-indolyl phosphate (NBT/BCIP) solution at room temperature. Samples were mounted in $80 \%$ glycerol.

\section{Coimmunoprecipitation and Western blotting}

Males (41-d-old) were homogenized in $400 \mu$ of lysis buffer. The protein extract was then pre-cleaned using protein G Sepharose 4 Fast Flow (GE Healthcare, catalog \#17-061801) and aliquoted for coimmunoprecipitation with the corresponding antibody [anti-CCB, anti-Rab4 (BD Biosciences, catalog \#610888; RRID:AB_398205), anti-Rab11 (BD Biosciences, catalog \#610656; RRID:AB_397983), anti- $\alpha$-Tubulin (DSHB, catalog $\# 12$ G10 anti- $\alpha$-Tubulin; RRID:AB_1157911), anti- $\beta$-Tubulin (DSHB, catalog \#E7 anti- $\beta$-Tubulin; RRID:AB_2315513), and anti- $\beta$-Actin (Abcam, catalog \#ab6276; RRID:AB_2223210) at $\sim 10 \mathrm{ng} / \mathrm{ml}]$. Upon washing, immunoprecipitated proteins were eluted by boiling in NuPAGE LDS sample buffer (Bio-Rad, catalog \#456-1083) and detected by Western blot using either the anti-CCB or anti-Rab11 antibody. Following PAGE and transfer, membranes were probed against rabbit monoclonal anti-CCB antibody (1:1000) or mouse monoclonal anti-Rab11 antibody (1:1000), followed by HRP-conjugated secondary antibody.

\section{Reporter visualization and immunostaining}

Immunostaining for CCB in whole mount brains from larvae and adults was as follows; brains were dissected in cold PBS, and then fixed for 30 min in cold $4 \%$ paraformaldehyde in PBST ( $0.5 \%$ Triton X-100 in PBS). Fixed samples were washed and then blocked in $1 \%$ normal goat serum for $2 \mathrm{~h}$ at $4 \mathrm{C}$. Primary antibody, monoclonal rabbit anti-CCB (1:10; GenScript; epitope sequence 33-VVESASRPQKYTFH-46), monoclonal mouse anti-Rab11 (1:10; BD Biosciences) or monoclonal mouse antiRab4 (1:10; BD Biosciences) were incubated overnight at $4^{\circ} \mathrm{C}$. Brains were then washed and incubated with the secondary fluorescent antibody AlexaFluor 488 or AlexaFluor 568 (1:400; Invitrogen) at room temperature for $2 \mathrm{~h}$. Specimens were mounted in VECTASHIELD (Vector Laboratories, catalog \#H-1000) and observed on a Leica TCS-SP5 equipped with a motorized inverted microscope (Leitz, DMIRE2). Serial optical sections $(1024 \times 1024$ pixels $)$ were taken at $1 \mu \mathrm{m}$ intervals using $40 \times$, $63 \times$ or $100 \times$ objectives.

\section{Axonal transport assay}

Axonal transport was assayed on the EB axonal branches of the Rneurons in the adult brain or neuromuscular junctions (NMJ) of larval motor neurons innervating muscle fibers 6 and 7 by expression of CD8:: GFP.

Axonal transport in the adult EB. Brains were dissected in cold PBS, and then fixed for $30 \mathrm{~min}$ in cold $4 \%$ paraformaldehyde in PBST $(0.5 \%$ Triton X-100 in PBS). After fixation, brains were washed and mounted in VECTASHIELD for imaging as described. Images were analyzed using ImageJ (NIH Image; RRID:SCR_003073) for quantification of signal intensity and pixel area over a region-of-interest (ROI) manually traced around the ellipsoid body.

Axonal transport in the larval NMJ. Wandering third instar larvae were dissected and prepared for immunohistochemistry (Martín-Peña et al., 2006). Immunostaining was performed as indicated in the previous section with the exception that all media was detergent-free to avoid penetration of the anti-CD8 antibody through the axonal membrane into the neurons. Primary antibodies anti-FasII (DSHB, catalog \#1D4 anti-

$\leftarrow$

(Figure legend continued.) $\quad(\boldsymbol{G}$; Kruskal-Wallis, $p=0.0052)$ to the axon terminals in the EB. Expression of the $c c b^{C D N A}$ does not modify the transport ratio for $\operatorname{CD} 8(C ;$ Kruskal-Wallis, $p=$ 0.1381 ) or Synaptobrevin $(\boldsymbol{H}$; Kruskal-Wallis, $p=0.5778) . \boldsymbol{E}$, $\boldsymbol{J}$, The total volume of the $E B$ is measured for each individual expressing either CD8::GFP $(\boldsymbol{E})$ or Synaptobrevin::GFP $(\boldsymbol{J})$. The volume of the EB in CD8 expressing flies ( $\boldsymbol{E}$; Kruskal-Wallis, $p>0.9999$ for flies coexpressing the $c C b^{R N A i}$ and $p=0.4518$ for flies coexpressing the $c\left(C^{C D N A}\right)$ or in Synaptobrevin-expressing flies ( $J$; Kruskal-Wallis, $p>0.9999$ for flies coexpressing the $c c b^{R N A i}$ and $p>0.9999$ for flies coexpressing the $c\left(b^{C D N A}\right)$ does not change. These results indicate that $c c b$ levels do not alter the volume or morphology of the R-neurons and, thus, the effects on CD8 and Synaptobrevin signals are specific of their axonal traffic. Scale bar, $10 \mu \mathrm{m}$. Error bars indicate SEM. ${ }^{* *} p<0.01 ; n=$ 6-14 per group.
Fasciclin II; RRID:AB_528235) and anti-CD8 (ThermoFisher Scientific, catalog \#MA1-90472; RRID:AB_1957420) were used at concentrations 1:500 and 1:500, respectively. Identical gain, offset, pinhole, and laser settings were used for experimental lines overexpressing or downregulating $c c b$ and controls. For quantification, pixel intensity was calculated within the same ROI delineated for each of the three channels, CD8 and GFP. We evaluated the amount of vesicle-associated CD8 (intracellular) through quantification of the GFP signal intensity from the construct CD8::GFP; and the portion of CD8 translocated to the cellular membrane by the signal from the anti-CD8 antibody (extracellular) in detergentfree conditions.

\section{Experimental design and statistical analysis}

For all experiments, controls and genetically matched experimental genotypes were performed in parallel. The experimental design ensured that all groups were balanced throughout the conducted experiments. The number of pUAS constructs was balanced for controls with the presence of a UAS-LacZ, compensating the UAS sites of experimental lines (RNAi, cDNA). Statistical analyses were performed using GraphPad Prism v5.0c. All data presented represent the mean \pm the SEM. One-way ANOVA nonparametric Kruskal-Wallis test followed by Dunns post hoc or Mann-Whitney comparisons were used throughout to analyze statistical significance. Wilcoxon test was used to analyze significance from zero.

\section{Results}

\section{The $c c b$ gene corresponds to the CG14579 transcription unit}

The $c c b$ mutations were originally characterized for their locomotor phenotype, which correlates with a severe anatomical phenotype in the central complex (Strauss and Heisenberg, 1993). It was later found that the mutation $c c b^{k s 145}$ prevents axons of R-neurons from crossing the midline and, thus, two independent, non-overlapping EBs form in each side of the midline (Renn et al., 1999). However, the molecular mechanism underlying this axonal phenotype remained unknown. To explore the molecular nature of $c c b$, we mapped the $c c b$ mutations by recombination, and located them between the cytological regions 19F1 and $19 \mathrm{~F} 2$ of the $\mathrm{X}$ chromosome (Fig. $1 A, B$ ). In this region, we identified the uncharacterized protein-coding transcription unit CG14579 as a candidate gene for the $c c b$ mutations (Fig. $1 A-C$; Tables 1,2,3). We next sequenced the coding region of CG14579 in the $c c b^{k s 127}$ and $c c b^{k s 145}$ alleles and compared them to the sequence we obtained from the wild-type Berlin, the parental strain where these mutant alleles had been induced. The coding region of CG14579 was intact in the $c c b^{k s 127}$ and $c c b^{k s 145}$ alleles; however, the lower expression of CG14579 in heads from flies carrying either $c c b$ allele (Fig. 1D) indicated that these mutations could affect regulatory regions rather than coding regions. These results pointed to CG14579 as the target of the regulatory mutations in $c c b$.

Given the expression phenotype observed in heads of $c c b$ mutant flies, we analyzed CG14579 distribution by in situ hybridization on cryosections of adult heads. Sections from Berlin control flies showed strong staining in neuron somata throughout the brain when incubated with the antisense probe (Fig. $1 G$ ), whereas negative control sections incubated with the sense probe did not produce positive staining (Fig. $1 H$ ). Sections from flies carrying either $c c b$ mutation showed no staining when incubated with the antisense probe (Fig. $1 I, K$ ) or with the sense probe (Fig. $1 J, L$ ). Then, to assess protein production in these mutants, we used a monoclonal antibody generated against the $\mathrm{N}$-terminal region of the protein (33-VVESASRPQKYTFH-46; Fig. 1C; Table 3). The antibody was validated in neuron somata of the adult and found virtually no CCB protein in brains of mutant flies (Fig. $1 F$ ). Overall, these data indicated that the mutations $c c b^{k s 127}$ and $c c b^{k s 145}$ 

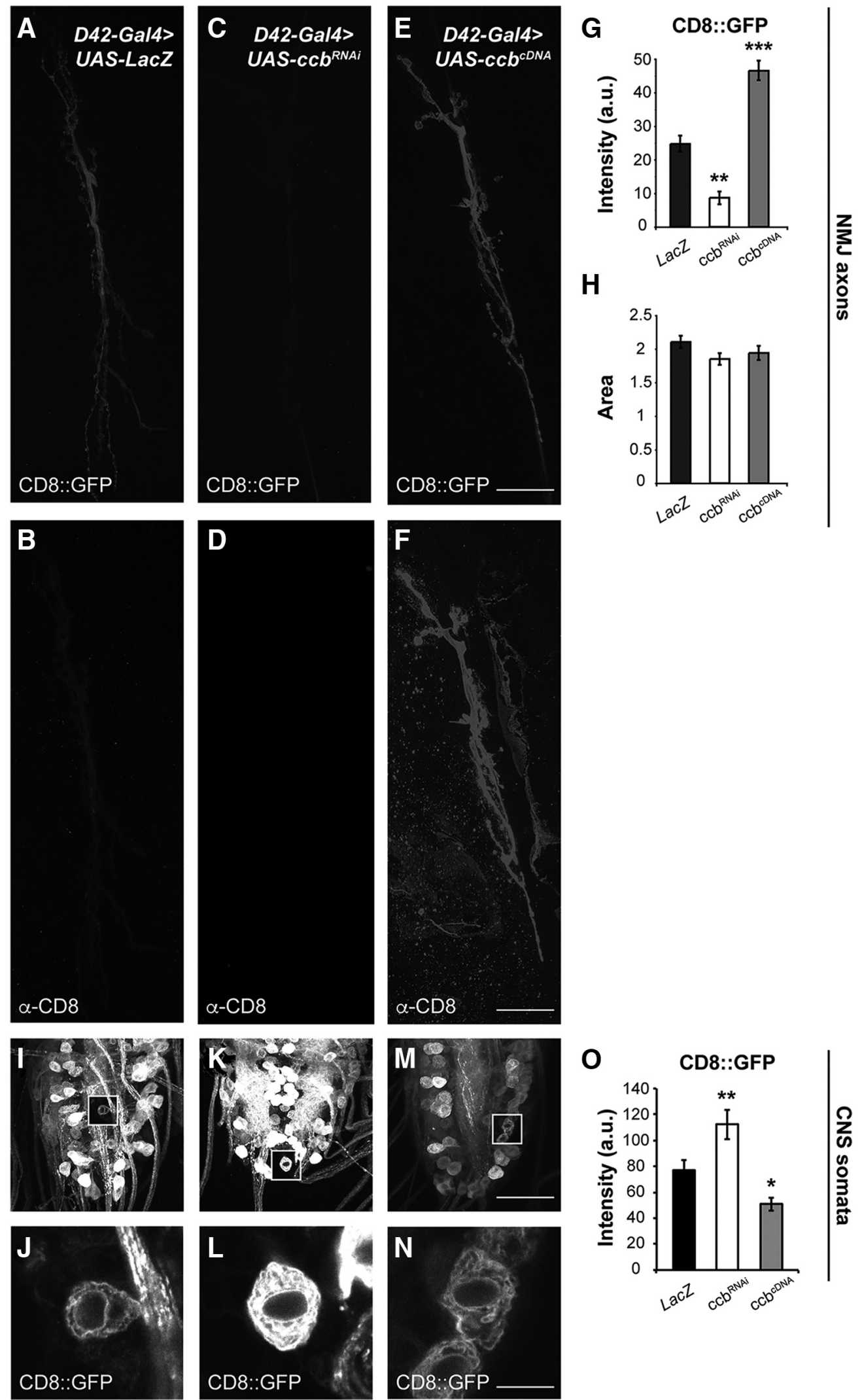

Figure 5. CCB-dependent vesicle-based axonal transport in larval motor neurons. $A-F$, NMJ of larval motor neurons innervating muscle fibers 6 and 7 . Vesicular CD8::GFP accumulated at the

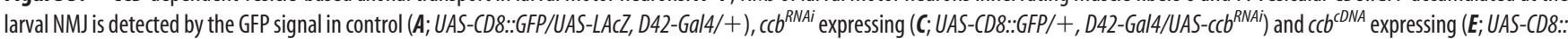
GFP/+, D42-Gal4/UAS-cC $C b^{C D N A}$ ) animals. Membrane-translocated CD8 is detected by the $\alpha$-CD8 antibody in the cellular membrane of control (B), $c b^{R N A i}(\boldsymbol{D})$, and $c c b^{C D N A}(\boldsymbol{F})$-expressing animals. $\boldsymbol{M}$, Quantification of the vesicle-based axonal transport of CD8::GFP to the NMJ in control, $c c b^{R N A i}$ and $c C b^{C D N} A$-expressing animals. Expression of $c c b^{R N A i}$ significantly reduces axonal transport of $C D 8$ (Kruskal-Wallis, $p=0.0023$ ), while ccb overexpression increases axonal transport of CD8 (Kruskal-Wallis, $p=0.0003$ ). N, NMJ area measured as the fluorescence signal for each genotype shown in $\boldsymbol{M}$. The size of the NMJ remains invariable among genotypes. $\boldsymbol{I}-\boldsymbol{N}$, Accumulation of CD8::GFP in the ventral ganglia of the larval CNS. Vesicular CD8::GFP accumulated in somata of the SNC motor neurons is detected by the GFP signal for control $(\boldsymbol{I}), c\left(b^{R N A i}(\boldsymbol{K})\right.$, and $c c b^{C D N A}(\boldsymbol{M})$ expressing animals. White squares show single-plane confocal images from regions (Figure legend continues.) 
abolish the ubiquitous expression of the CG14579 gene in the Drosophila brain, confirming the regulatory character of the $c c b$ alleles.

It then seemed plausible that the lack of $c c b$ expression in the brain was the cause of the morphological phenotypes previously observed in the central complex (Fig. $2 A-C$ ). To unequivocally validate the identity between the $c c b$ mutations and the CG14579 gene, we ubiquitously expressed the CG14579 cDNA, using the tubulin driver (LL7-Gal4), in an otherwise mutant background for $c c b\left(c c b^{k s 127} ; U A S-c c b^{c D N A} ; L L 7-G a l 4\right)$. Expression of the $c c b^{c D N A}$ rescued the morphological phenotype previously described in the EB (Fig. 2D-F). Furthermore, selected expression of the $c c b^{R N A i}$ in R-neurons recapitulated the developmental phenotype of $c c b$ in the EB (Fig. 2G-I). These data demonstrated that $c c b$ is required and sufficient for proper formation of the axonal pattern of R-neurons in the EB.

\section{CCB regulates vesicle-based axonal transport in R-neurons of the EB}

Given the morphological phenotype in the EB, because of the lack of CCB, we reasoned that CCB might be involved in (1) axonal growth or (2) axonal guidance, either being a signaling molecule that regulates axonal pathfinding itself or regulating the transport of signaling molecules. Therefore, we implemented an axonal transport assay (Murthy et al., 2003) to further investigate the role of CCB in vesicular transport of proteins along axons of R-neurons in adult brains (Figs. $2 G-L, 4$ ). In these neurons, we assessed the transport of CD8::GFP (Figs. 2G-L, 4A-E) and Synaptobrevin::GFP (Fig. $4 F-J$ ) to axon terminals in the EB. To downregulate $c c b$ expression, we designed a $c c b^{R N A I}$ contruct (Fig. 3-1, available at https://doi.org/10.1523/JNEUROSCI.091518.2019.f3-1) that was proven functional when panneuronally expressed in neurons (Fig. 3). Expression of the $c c b^{R N A i}$ in R-neurons led to a significant reduction of CD8::GFP (Figs. 2G$L, 4 B, D$ ) and Synb::GFP (Fig. 4G,I) in the EB, whereas expression of the $c c b^{c D N A}$ did not elicit significant changes in the transport rate of CD8::GFP (Fig. 4C,D) nor Synb::GFP (Fig. 4H,I). The phenotypic effect of downregulating $c c b$ in R-neurons resulted stronger when using the c507-Gal4 driver (Fig. $2 G-L$ ) due to the fact that the 796-Gal4 (Fig. $4 B, D$ ) is a hypermorph allele of $c c b$ (Fig. 1E). Interestingly, GFP intensity in the somata of R-neurons was inversely proportional to that of the CD8::GFP detected in the EB (Fig. 2J-L). Further, the size of the EB remained constant among groups (Fig. $4 E, J$ ). These results indicated that $\mathrm{CCB}$ is involved in vesicle-based axonal transport of CD8 and Synaptobrevin.

\section{CCB regulates axonal transport of selected synaptic proteins}

Next, we further investigated the role of CCB in the transport of additional synaptic proteins in larval motor neurons using the original axonal transport assay (Murthy et al., 2003). In this assay, we monitored axonal transport of specific cargos from the soma

\section{$\leftarrow$}

(Figure legend continued.) of interest containing individual cell bodies. J, $L, N$, Magnification of the insets in $\boldsymbol{I}, \boldsymbol{K}, \boldsymbol{M}$ for the corresponding genotypes: control (J; UAS-CD8::GFP/UAS-LACZ,

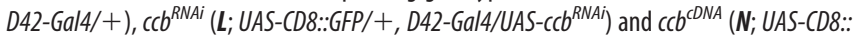
GFP/+, D42-Gal4/UAS-cCb $\left.b^{\text {CDNA }}\right)$. 0, Quantification of the GFP signal $(\boldsymbol{I}, \boldsymbol{K}, \boldsymbol{M})$ shows significant retention of CD8::GFP in the soma of motor neurons expressing the $\mathrm{cCb}^{R N A i}(\boldsymbol{K} ;$ Kruskal-Wallis, $p=0.0064)$, and a reduction of the GFP signal in somata of animals expressing the $c C b^{C D N A}$ in larval motor neurons ( $\boldsymbol{M}$; Kruskal-Wallis, $p=0.0138$ ). Error bars indicate SEM. ${ }^{*} p<0.05$, ${ }^{* *} p<0.01,{ }^{* * *} p<0.001 ; n=5-10$ per group. Scale bars: $A-F, 12 \mu \mathrm{m} ; I, K, M, 48 \mu \mathrm{m} ; J, L$, $N, 8 \mu \mathrm{m}$. of larval motor neurons to synaptic boutons in the NMJ. Specifically, we expressed GFP-tagged CD8 in motor neurons using the D42-Gal4 to monitor its transport to axon terminals and, simultaneously, the proportion that is translocated to the plasma membrane. The transport of intracellular CD8 along the axon was measured by the GFP attached to the receptor, whereas CD8 translocation to the surface of the cellular membrane was detected with the anti-CD8 antibody incubated in a detergent-free medium, thus preventing the antibody to penetrate inside the cell.

We focused on the axon terminals innervating muscle fibers 6 and 7 on the third abdominal segment of third instar larvae. In control animals we could observe a relatively intense GFP signal along the axon terminal (Fig. $5 A$ ), whereas the signal from CD8 staining was considerably less intense (Fig. 5B). Expression of the $c c b^{\mathrm{RNAi}}$ driven by $D 42-$ Gal4 abolished almost completely the transport of CD8 to the axon terminals (Fig. $5 C$ ) and, consequently, its translocation to the cell membrane (Fig. 5D). On the contrary, overexpression of $c c b$ in these larval motor neurons increased the amount of CD8 in axon terminals (Fig. 5E) and CD8 translocation to the surface of the plasma membrane (Fig. $5 F$ ). These results suggested that the translocation phase was limited by the ratio of transport up to the axon terminals (see quantification, Fig. 5G,H). The GFP signal in the cell body of these motor neurons was inversely proportional to that of the axons (Fig. 5I-O). Further, these data implied that $c c b$ is involved in protein trafficking during the initial phases of the transport process.

Following the same experimental procedure, we investigated the transport rate of other proteins involved in different aspects of synaptogenesis: Rab11, FasII, and syntaxin. The transport of GFP-tagged Rab11 and FasII was significantly reduced when coexpressing the $c c b^{R N A i}$ (Fig. $6 A, B$ ). However, $c c b$ overexpression did not lead to any significant increase or decrease in the transport of Rab11 or FasII to the axon terminal (Fig. $6 A, B$ ). It is plausible that loading of these two cargos to the CCB-dependent system is limited by other independent mechanisms. On the other hand, neither expression of the $c c b^{R N A i}$ nor overexpression of the $c c b$ led to any change in the amount of Syntaxin accumulated in the NMJ (Fig. 6C), suggesting that a different transport system mediates Syntaxin trafficking. Finally, although up and downregulation of $c c b$ selectively affected the transport rate of specific proteins, the size and morphology of the axon terminals remained intact for most genotypes (Fig. $6 \mathrm{Bv}, \mathrm{Cv}$ ). Co-overexpression of Rab11 with the $c c b^{\mathrm{RNAi}}$, however, significantly decreased the size of the NMJ (Fig. $6 \mathrm{Av}$ ). In fact, overexpression of Rab11 in the NMJ has been previously reported to affect axonal growth (Khodosh et al., 2006). Together, these data indicate that $c c b$ is involved in axonal transport of specific cargos including FasII and synaptobrevin, and suggest the presence of an independent transport system for other cargos like syntaxin.

\section{CCB colocalizes with Rab11}

Once we confirmed the general role of CCB in axonal transport, in two neuronal populations, we set to explore the molecular mechanism that CCB uses for protein trafficking. We used the anti-CCB antibody to examine the subcellular location of the CCB protein. The antibody revealed a perinuclear staining and a punctate pattern throughout the cytoplasm and the plasma membrane, compatible with vesicles and other membranous organelles (Fig. 7A-C; Table 3). This pattern was present in cortical neurons of the adult brain (Fig. $7 A$ ), as well as in sensory neurons $(7 B)$ and projection neurons $(7 C)$ of the larva. 

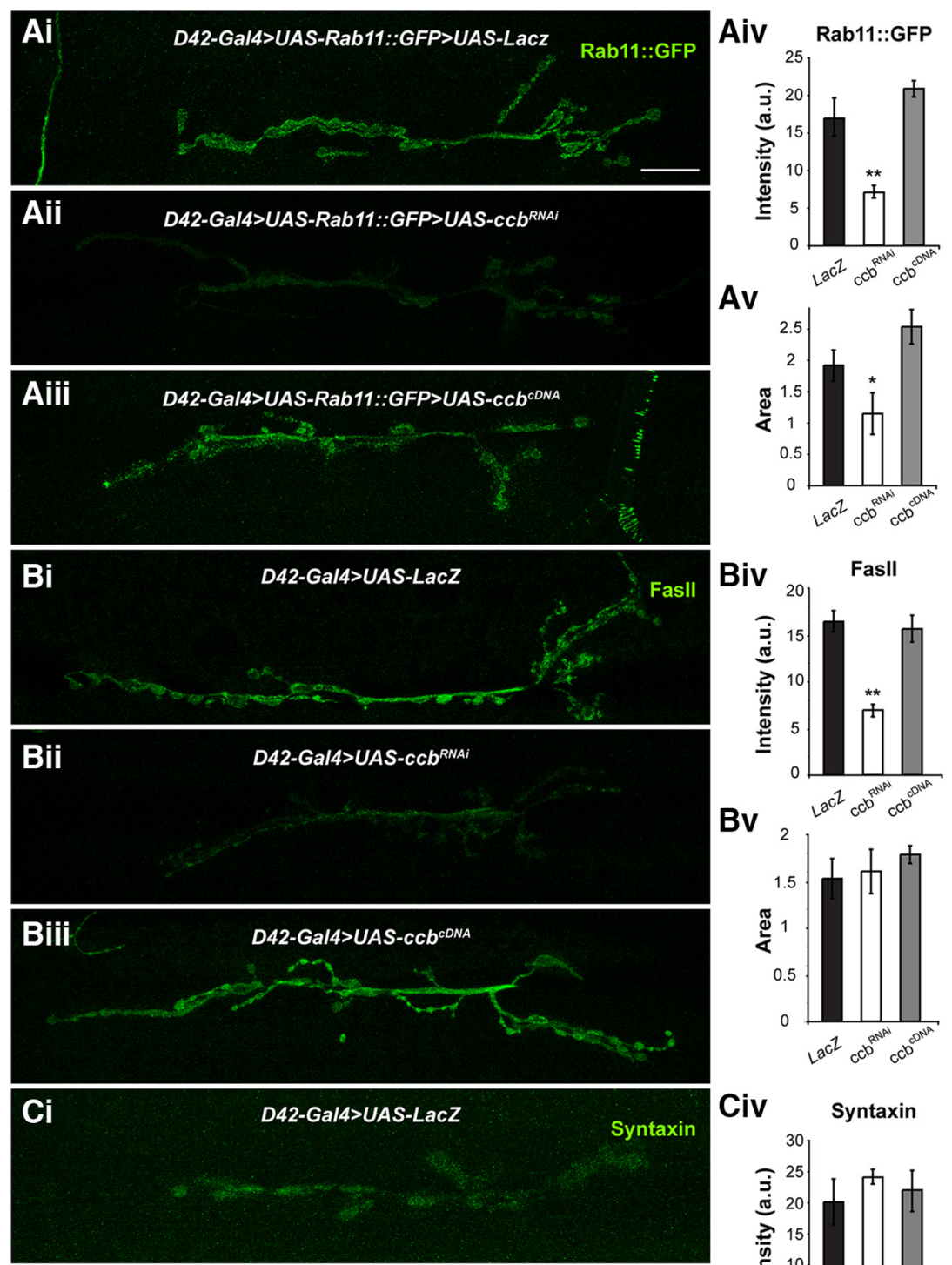

\section{Civ}
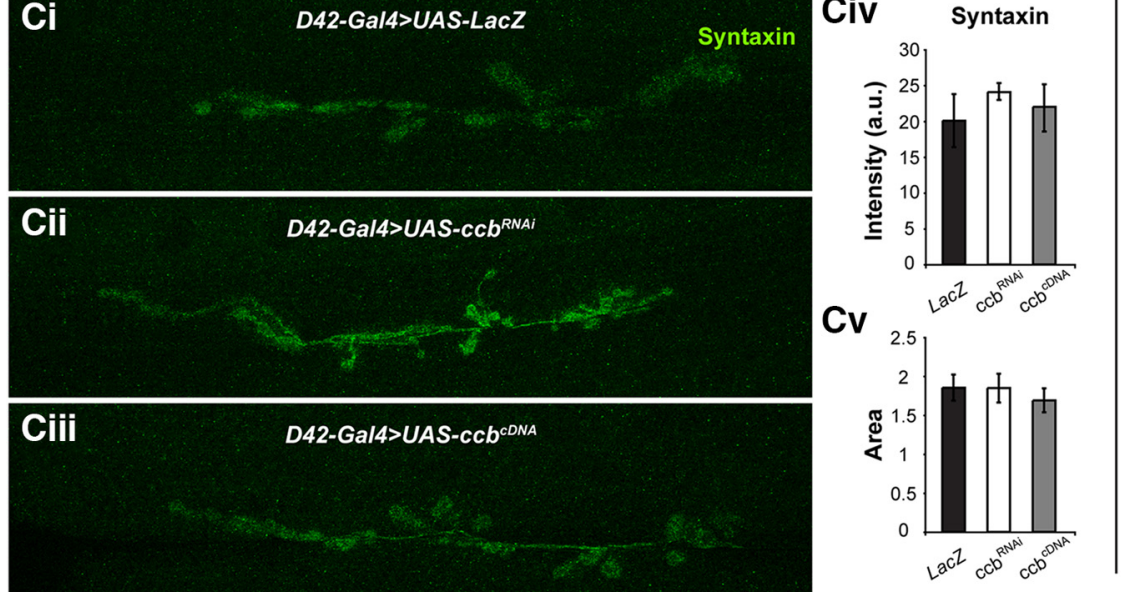

Figure 6. Axonal transport of selected synaptic proteins to the larval NMJ. NMJ of larval motor neurons innervating muscle fibers 6 and 7 for controls (Ai, Bi, $\boldsymbol{C}$ ), and animals expressing the cCb $b^{R N A i}$ (Aii, Bii, Cii) or the $c_{C} b^{\text {CDNA }}$ (Aiii, Biii, Ciii) with the Gal4 driver D42. A, The GFP signal reveals the amount of Rab11 transported to the NMJ (Rab11::GFP) for the corresponding three genotypes (Ai-Aiii). B, C, Immune signal for Fasciclin II and Syntaxin detecting the amount of protein accumulated at the NMJ for each corresponding genotype (Bi-Biii, $\boldsymbol{C}$-Ciii). Note the reduced amount of Rab11 and Fasll, but not of Syntaxin due to downregulation of ccb in motor neurons. Aiv, Biv, Civ, Quantification of the axonal transport to the NMJ for Rab11, Fasll, and syntaxin in control, $c c b^{R N A i}$-expressing, and $c c b^{C D N A}$-expressing animals. Expression of $c c b^{R N A i}$ significantly reduces axonal transport of Rab11 (Kruskal-Wallis, $\left.p=0.0024\right)$ and Fasll (Kruskal-Wallis, $p=0.0023$ ) but not syntaxin (Kruskal-Wallis, $p=0.1806$ ). ccb overexpression does not modify the transport of Rab11 (Kruskal-Wallis, $p>0.9999$ ), Fasll (Kruskal-Wallis, $p=0.5356$ ) or syntaxin (Kruskal-Wallis, $p=0.8370) . A v, B v, C v, N M J$ area measured as the fluorescence signal for each genotype shown in Aiv, Biv and Civ. Despite the significant changes observed in the ratio of axonal transport for specific synaptic proteins upon manipulation of the ccb levels, the size of the NMJ remains invariable except when Rab11 is downregulated (Kruskal-Wallis, $p=0.0154)$. Error bars indicate SEM. ${ }^{*} p<0.05,{ }^{* *} p<0.01 ; n=5-10$ per group. Scale bar, $12 \mu \mathrm{m}$.

We used these larval projection neurons to further investigate the subcellular compartment where CCB is located (Fig. $7 D, G, J, M$ ). We tested several Rab proteins associated with membranous compartments and known to mediate vesicle- and motor protein-based transport. Colocalization studies with Rab4 (Fig. 7E) showed a close proximity of the two proteins in most cases but not a complete overlap (Fig. 7F). Rab11 (Fig. 7H), however, exhibited a perfect overlap with the distribution of CCB in these neurons (Fig. 7I), 

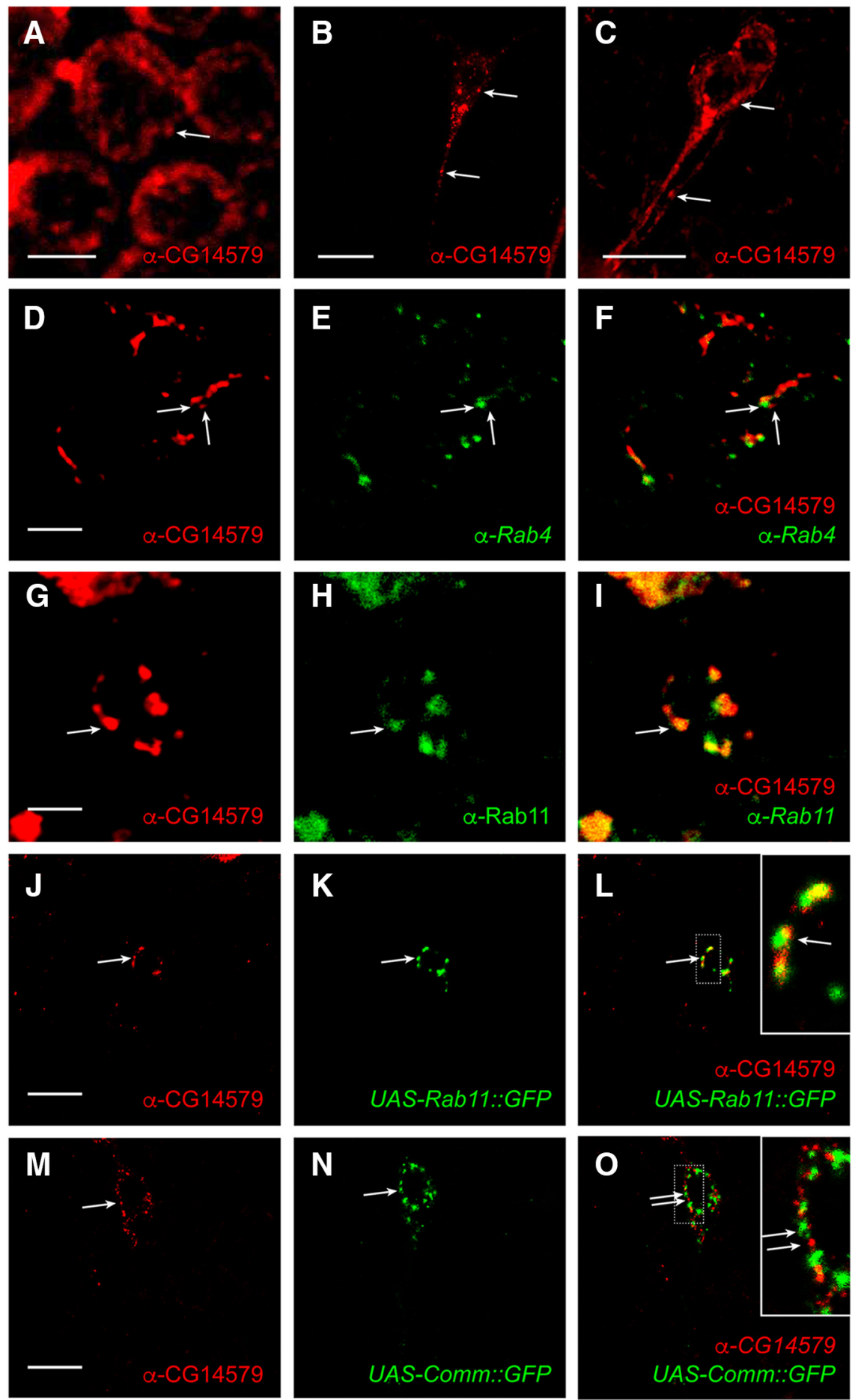

$\mathbf{P}$

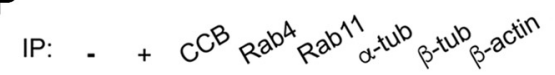

Q

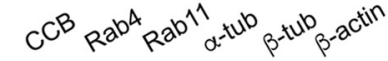
$\mathrm{CCB} \rightarrow$

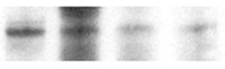

Figure the adult brain $(A)$ sensory neurons from third instar larva $(B)$ and $B$-neuron progenitors from the larval brain $(O$. Note the punctate pattern in the cytoplasm of all these cells (arrows). $D-I$, Distribution of the endogenous protein in larval brains stained against $C(B(\boldsymbol{D}, \boldsymbol{G}), \operatorname{Rab} 4(\boldsymbol{E})$, and Rab11 $(\boldsymbol{H})$. The immune signal shows that $C(B)(\boldsymbol{D})$ and $\operatorname{Rab} 4(\boldsymbol{E})$ do not overlap in their subcellular localization $(\boldsymbol{F})$. However, both proteins are adjacent to each other $(\boldsymbol{F})$. The antibody staining shows a perfect overlap in the subcellular distributions of the endogenous $C(B)(G)$ and $\operatorname{Rab} 11(\boldsymbol{H})$ proteins. $I$, The subcellular localization of both proteins matches in every compartment. J-0, Larval brains stained with the $\alpha$-CCB antibody $(\boldsymbol{J}, \boldsymbol{M})$ and expressing Rab11::GFP $(\boldsymbol{K})$, or commissureless:::GFP $(\boldsymbol{N})$ in the larval progenitors of the R-neurons under the control of the Gal4-796. The distributions of CCB $(J)$ and GFP-tagged Rab11 ( $K$ ) overlap in larval projection neurons ( $\boldsymbol{L}$ ). High-magnification images reveal a nearly perfect overlap ( $\boldsymbol{L}$, inset). $M-\mathbf{0}$, CCB distribution is observed in punctate patterns in R-neurons $(\boldsymbol{M})$ and exhibits a similar distribution to that of commissureless::GFP $(\boldsymbol{M})$, suggesting that CCB and Rab11 belong to the same membrane domain.

Next, we used the 796-Gal4 line to stain larval brains against CCB (Fig. $7 \mathrm{~J}, \mathrm{M}$ ) for two main reasons: (1) the 796Gal4 drives expression in R-neurons of the EB (Martín-Peña et al., 2014), thus allowing visualization of fluorescentlytagged proteins in a small set of neurons (rather than in every neuron of the brain); and (2) CCB staining of larval brains reveals preferential expression of $c c b$ in R-neurons, which facilitates colocalization assays with single-cell resolution. This preferential expression is likely due to the P-element insertion site in the $c c b$ locus of 796-Gal4 flies that significantly increases CG14579 expression (Fig. 1E). We expressed a GFP-tagged Rab11 in these R-neurons and, after anti-CCB staining (Fig. 7J), observed a pattern strikingly similar to that of CCB $(7 \mathrm{~J}, K)$. In fact, the distribution of CCB and Rab11 overlapped in somato-axonic regions of the R-neurons (Fig. 7L).

Last, inspired by the abnormal axonal guidance phenotype detected in $c c b$ mutants (Fig. 2A-C), we used the 796-Gal4 driver to express GFP-tagged commissureless (COMM; Tear et al., 1996), a transmembrane protein that regulates the transport of the receptor $\mathrm{ROBO}$ to the plasma membrane in axonal growth cones of commissural neurons. COMM was distributed in a similar, albeit not identical, pattern to that of CCB (Fig. $7 M, N$ ), indicating that both proteins are located within the same subcellular compartment. However, their distribution did not overlap (Fig. 7O). Together, CCB is located in the soma and initial segment of the axon, where it colocalizes with Rab11. The distribution of CCB and its association with Rab11, which is part of the protein transport system that delivers specific cargos to synapses, positions CCB as a potential member of the same trafficking system.

which is consistent with proteins associated with vesicular organelles; however, no overlap is found between $\triangle(B$ and COMM (0). High-magnification images confirm the lack of overlap ( $\mathbf{0}$, inset). $\boldsymbol{P}, \mathbf{Q}$, Coimmunoprecipitation assays (lane 1 : agarose beads alone, no antibody; lane 2: protein extract preantibody immunoprecipitation). Coimmunoprecipitation of CCB (P) and Rab11 (Q) with CCB (lane 3), Rab4 (lane 4), Rab11 (lane 5), $\alpha$-tubulin (lane 6), $\beta$-tubulin (lane 7), or $\beta$-actin (lane 8). Scale bars: $\boldsymbol{A}, 3 \mu \mathrm{m} ; \boldsymbol{B}, \mathbf{C}, 12 \mu \mathrm{m} ; \boldsymbol{D}-\mathbf{I}, 6 \mu \mathrm{m} ; \boldsymbol{J}-\mathbf{0}$, $12 \mu \mathrm{m}$. 


\section{CCB coimmunoprecipitates with Rab11}

To investigate the potential interaction between CCB and Rab11, we performed coimmunoprecipitation assays with protein extracts from adults. We first detected CCB in protein extracts immunoprecipitated with CCB itself, Rab4, Rab11, and $\beta$-Actin, whereas it was undetectable, or at very low levels, in extracts immunoprecipitated with $\alpha$-tubulin or $\beta$-tubulin (Fig. $7 P$ ). On the other hand, the reverse experiment showed the presence of Rab11 in immunoprecipitates with CCB, Rab4, Rab11, and $\alpha$-actin, but not with $\alpha$-tubulin or $\beta$-tubulin (Fig. 7Q). Although these results suggested an interaction between CCB and Rab4, it is possible that a linker could mediate this association. This alternative would be consistent with the adjacent, but nonoverlapping, localization observed for these two proteins (Fig. $7 F$ ), hence, there would be no direct physical interaction between $\mathrm{CCB}$ and Rab4. Together, these data position CCB within the Rab4/Rab11 domain of slow recycling endosomes (Sönnichsen et al., 2000). These results support a transport system that includes CCB, Rab4, Rab11, and actin, while excluding the main component of microtubules, tubulin.

\section{Rab11 is downstream of CCB for axonal branching regulation} in R-neurons of the EB

Based on the axonal growth phenotypes of Rab11 (Khodosh et al., 2006) and CCB (Renn et al., 1999), we further explored their interaction in the context of axonal branching within the EB. To address this question, we used two Rab11::YFP constructs that enabled us to visualize the axonal projections of R-neurons while manipulating Rab11 activity and the levels of $c c b$. Expression of a constitutively active form of Rab11 (Q70L) led to an increased axonal branching of the R-neurons in the EB (Fig. $8 A$ ). This effect was significantly marked in the branching domain of $\mathrm{R} 2$ neurons within the outer ring (Fig. 8A; Martín-Peña et al., 2014). On the contrary, expression of a dominant-negative form of Rab11 $(\mathrm{S} 25 \mathrm{~N})$ reduced the branch density in the EB (Fig. $8 B$ ), being virtually absent in the $\mathrm{R} 2$ domain of the outer ring. Interestingly, coexpression of the $c c b^{R N A i}$ with the constitutively active form of Rab11 did not exhibit any significant change in the EB branching (Fig. $8 C, E$ ). However, $c c b^{R N A i}$ coexpression with the dominantnegative construct triggered a more pronounced branch reduction than $R a b 11^{D N}$ alone (Fig. $8 D$ ), as measured by YFP intensity (Fig. $8 E$ ). The fact that knockdown of $c c b$ could not prevent the branching activity of constitutive Rab11 positions Rab11 downstream of CCB, within this axonal transport system. This is consistent with the lack of axonal transport enhancement upon CCB overexpression (Figs. 4, 6); likely hampered by downstream components such as Rab11.

\section{$c c b$ Interacts with the actin nucleator factor spire}

The interaction of actin with various components of this network (Fig. $7 P, Q$ ), including CCB and Rab11, and the physical interaction reported by yeast two-hybrid (Giot et al., 2003) between $c c b$ and spir (http://thebiogrid.org/59376) along with the matching of their expression profiles led us to question whether these two molecules were part of the trafficking network investigated here. We observed that axonal transport of CD8::GFP to NMJs of motor neurons expressing the spire ${ }^{R N A i}$ was significantly impaired (Fig. $9 A-C$ ), retaining the GFP-tagged CD8 in the corresponding somata (Fig. $9 D-F$ ). These results mimicked the phenotype of $c c b$ in larval motor neurons (Fig. 5) and, thus, indicate that both CCB and SPIRE regulate axonal transport.

To test the possible functional relationship between the two genes, we combined the $c c b$ mutants, $c c b^{k s 127}$ and $c c b^{k s 145}$, sepa-
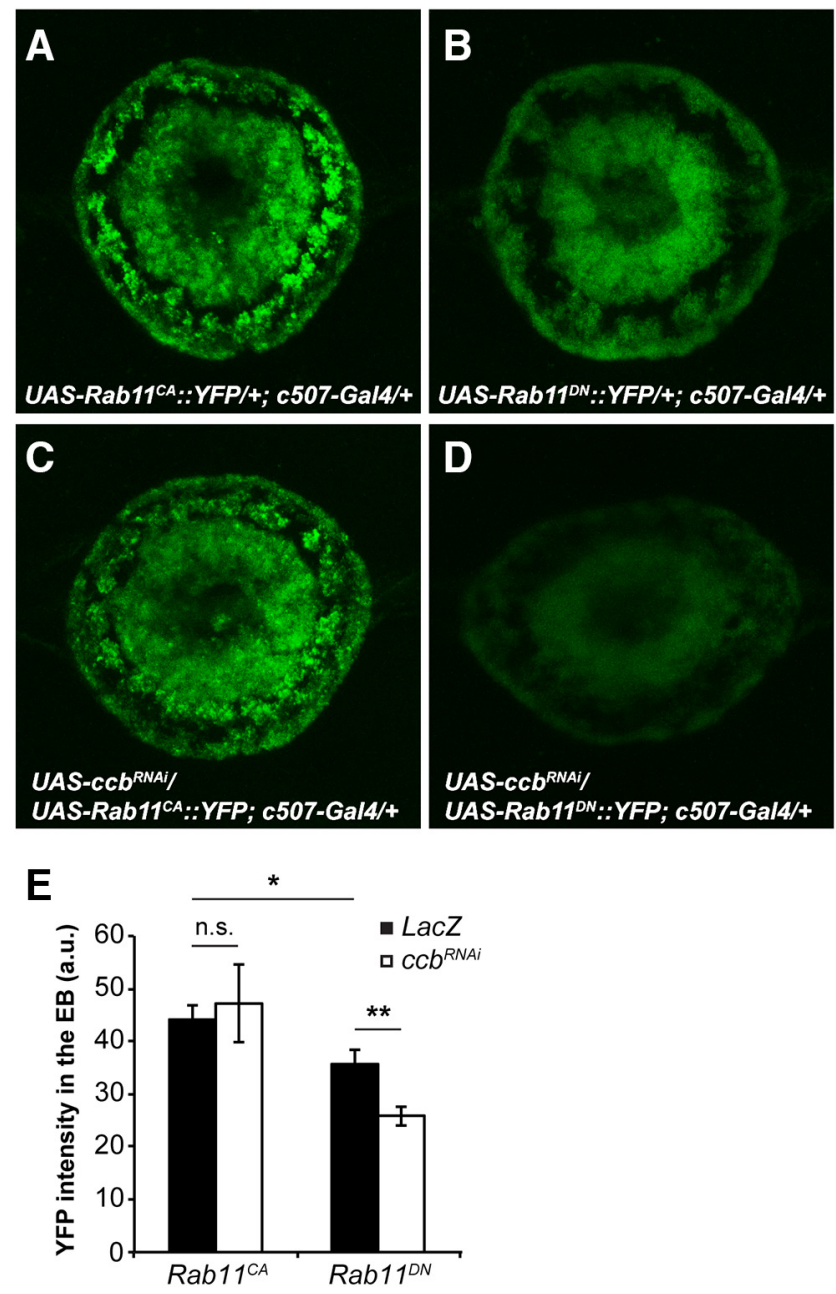

Figure 8. Rab11 regulates R-neuron axonal branching downstream of CCB. Axonal branches of $\mathrm{R}$-neurons innervating the adult $\mathrm{EB}$. Flies expressing either the constitutively active form of

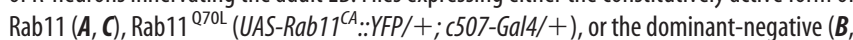
D), Rab11 ${ }^{\text {S25N }}$ (UAS-Rab11 ${ }^{\text {DN.:YFP/+; } 507-G a l 4 /+)}$, alone $(\boldsymbol{A}, \boldsymbol{B})$ or in combination with the $c C b^{R N A i}(\boldsymbol{C}, \boldsymbol{D})$ under the control of the $C 507-G$ al4 driver. $\boldsymbol{E}$, Quantification of the YFP signal in the EB. YFP pixel intensity was significantly lower in R-neurons expressing the dominant-negative form of Rab11 compared with the constitutively active one (Kruskal-Wallis, $p=0.0185$ ). This effect was enhanced when coexpressing the $c b^{R N A i}$ and Rab11 ${ }^{D N}$ (Kruskal-Wallis, $p=0.0079$ ); however, downregulation of $c c b$ did not affect Rab11 ${ }^{C A}$-induced branching in the EB (KruskalWallis, $p=0.8999$ ). Scale bar, $30 \mu \mathrm{m}$. Error bars indicate SEM. ${ }^{*} p<0.05,{ }^{* *} p<0.01 ; n=$ 8-12 per group. n.s. $=$ not significant.

rately with a spire mutant, spir ${ }^{1}$, and analyzed the resulting phenotypes. The homozygous condition for spir ${ }^{1}$ caused $70 \%$ lethality compared with heterozygous siblings. The combination of either $c c b^{k s 127}$ or $c c b^{k s 145}$ with $s p i r^{1}\left(c c b^{k s 127}\right.$; $s p i^{1 /+}$ or $c c b^{k s 145}$; $\left.s p i r^{1 /+}\right)$, significantly increased the lethality observed for $s p i r^{1}$ alone $\left(c c b^{+}\right.$; spir $\left.{ }^{1 /+}\right)$ by $20-30 \%$. Then, we evaluated the morphological consequences of this potential genetic interaction. We analyzed the EB morphology of males carrying $\operatorname{spir}^{1}$ in combination with either of the $c c b$ mutations $\left(c c b^{k s 127} ; \operatorname{spir}^{1 /+}\right.$ or $c c b^{k s 145}$; spir $\left.{ }^{1 /+}\right)$. spir ${ }^{1}$ alone did not display any morphological alteration in the EB; however, its combination with the $c c b$ mutants increased the penetrance of the $c c b$ phenotype in the EB (Fig. $9 G$ ). The penetrance of the strongest phenotype (split EB) on a spir ${ }^{1}$ background increased $30 \%$ in $c c b^{k s 127}$ flies and $15 \%$ in $c c b^{k s 145}$ flies, bringing the penetrance up to $100 \%$ (Fig. 9G). These data represent genetic evidence of a synergistic effect between $\mathrm{CCB}$ and SPIRE and suggest that both proteins work together for 

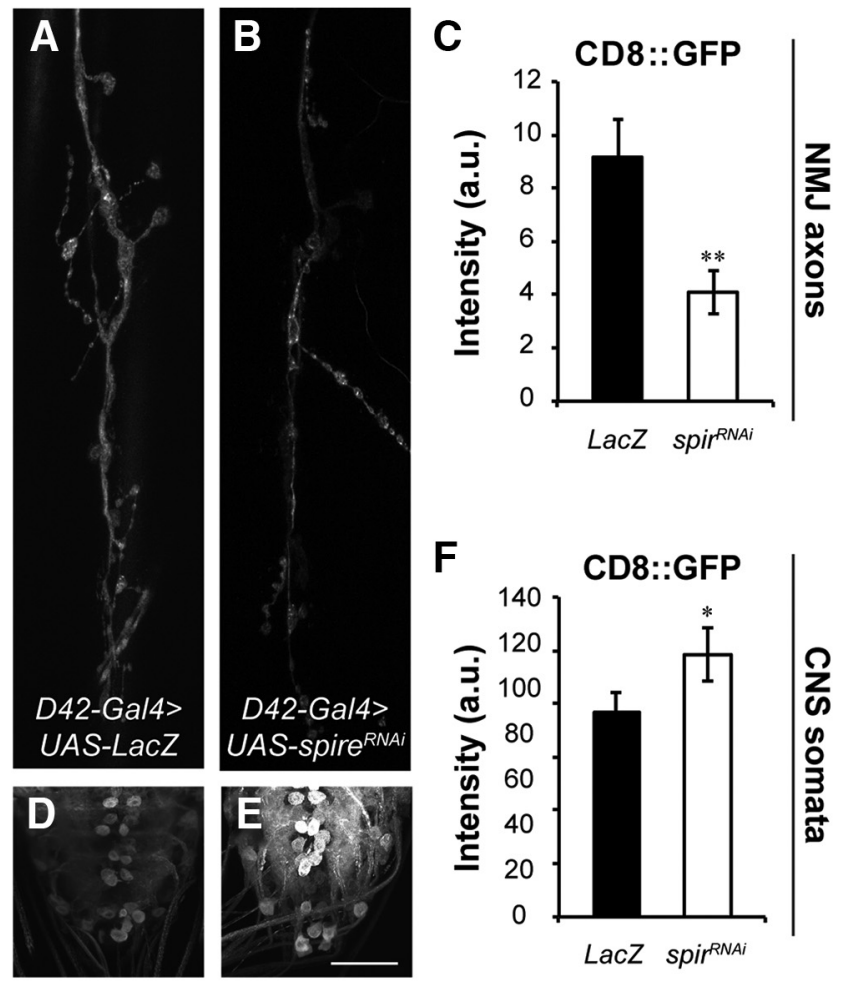

G

$\square$ circular $\square$ open $\square$ split

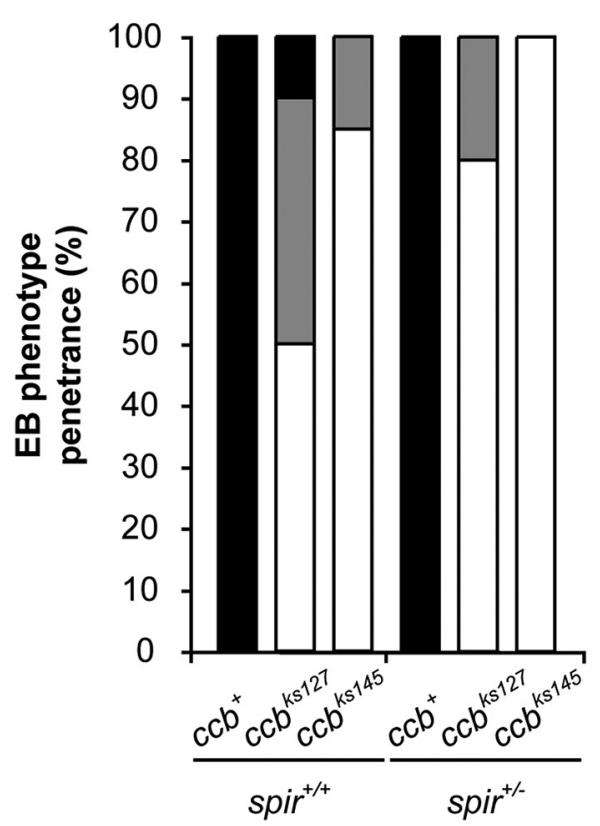

Figure 9. spire loss of function impairs axonal transport and enhances the $c c b$ phenotype in the EB. $\boldsymbol{A}, \boldsymbol{B}, \mathrm{NMJ}$ of larval motor neurons innervating muscle fibers 6 and 7 . Vesicular CD8::GFP accumulated at the larval NMJ is detected by the GFP signal for control ( $A$; UAS-CD8::GFP/UAS-

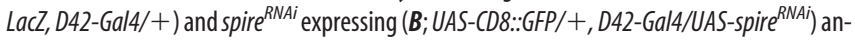
imals. $C$, Quantification of the GFP signal in $(\boldsymbol{A}, \boldsymbol{B})$ reveals a significant reduction of vesicle-based axonal transport of CD8::GFP to the larval NMJ in motor neurons expressing the spire ${ }^{R N A i}\left(C_{;}\right.$; Kruskal-Wallis, $p=0.0094)$. $\boldsymbol{D}, \boldsymbol{E}$, Accumulation of CD8::GFP in somata of the larval ventral ganglia. CD8::GFP accumulated in somata of the CNS motor neurons is detected by the GFP signal for control (D; UAS-CD8::GFP/UAS-LacZ, D42-Gal4/+) and spire ${ }^{\text {RNAi }}$ expressing (E; UAS-

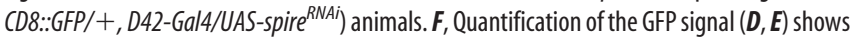
significant retention of CD8::GFP in the soma of motor neurons expressing the spire ${ }^{R N A i}(\boldsymbol{F}$; Kruskal-Wallis, $p=0.0263)$. G, Quantification of the $c c b$ phenotype in the ellipsoid body of wild-type and heterozygous mutant background for spire. Flies carrying the $c c b$ mutations proper axonal guidance. Because SPIRE is an actin nucleator factor and CCB/Rab11 coimmunoprecipitated with actin (Fig. $7 P, Q)$, it seems likely that the actin network could support axonal transport to growth cones and synaptic sites, to attain proper synaptic connectivity, synaptogenesis and, perhaps, synaptic plasticity underlying learning.

\section{Discussion}

To better understand the molecular mechanisms of protein trafficking, we investigated the role of CCB in axonal transport. We observed that $c c b$ is required for vesicle-based axonal transport of synaptic proteins in adult R-neurons of the EB and in larval motor neurons. The data showed that $\mathrm{CCB}$ regulates the trafficking of selected cargos, including CD8, Rab11, Synaptobrevin and FasII, but not Syntaxin. Of note, the role of adhesion molecules, including FasII (Yu et al., 2000; Kurusu et al., 2002; Fushima and Tsujimura, 2007), and other signaling molecules such as Rab11 (Khodosh et al., 2006) are key for axonal growth regulation. However, we focused on synaptic proteins because of the increasing evidence that proper axonal transport is intimately related to synaptic function. Further, CCB was observed in the soma and in the initial segment of the axon, where it colocalizes with Rab11. Immunoprecipitation assays demonstrated a molecular interaction of CCB with Rab11 and Actin. Furthermore, in the EB, the actin nucleator factor SPIRE elicited a synergistic effect over the $c c b$ mutant phenotype that prevents R-neuron axons from crossing the midline. We propose that both proteins are part of a selective traffic system that delivers cargos to the correct synaptic destination via the actin cytoskeleton within the Rab11-related domain of slow recycling endosomes.

The $c c b$ gene was initially characterized for its locomotor deficits (Strauss and Heisenberg, 1993) and was later found that the impaired locomotion was due to morphological defects preventing axonal crossing of the midline (Renn et al., 1999). However, the identity and molecular mechanism of this gene was never investigated. We identified the $c c b$ alleles, $c c b^{k s 127}$ and $c c b^{k s 145}$, as regulatory mutations that result in a loss of function of GC14579 (Fig. 1D). Although these regulatory regions could potentially modulate expression of adjacent genes, it is unequivocally evident that CG14579 is sufficient to rescue the morphological phenotype of $c c b$ in the EB (Fig. 2D-F). Moreover, CG14579 is required for proper axonal growth of R-neurons (Fig. 2G-I). CCB regulates axonal transport of synaptic proteins from the ER to synapses and, thus, it is plausible that this trafficking system regulates the transport of molecules required for the midline crossing of growing R-neuron axons. For instance, it has been previously described that COMM regulates transport of the receptor ROBO from the ER to the membrane surface (Tear et al., 1996; Georgiou and Tear, 2003). This COMMdependent transport system regulates the presence of $\mathrm{ROBO}$ in the surface of growth cones to determine whether axons cross the midline, and whether they then stay in the contralateral side or return to their side of origin. Specifically in the EB, semaphoring-1a and plexin

$\leftarrow$

$\left(c c b^{k s 127}\right.$ or $\left.c c b^{k 5145}\right)$ are grouped into three different categories based on the EB morphology, "circular" for flies with a morphologically normal EB, "open" for flies with an EB where the ventral region is not completed, and "split" for flies with two independent EBs, each of them located on opposite sides of the midline and formed only by axons from ipsilateral R-neurons. The proportion of each phenotype is calculated as the percentage of the total number of flies analyzed for each genotype. The data show a significant increase in the severity of these aberrations on a spire mutant background $\left(\right.$ spir $\left.^{1 /+}\right)$ compared with controls $\left(\right.$ spir $\left.^{+/+}\right)$. Scale bars: $\boldsymbol{A}, \boldsymbol{B}, 12 \mu \mathrm{m} ; \boldsymbol{D}, \boldsymbol{E}, 50 \mu \mathrm{m}$. Error bars indicate SEM. ${ }^{*} p<0.05,{ }^{* *} p<0.01 ; n=3(\boldsymbol{C}, \boldsymbol{F})$ and 25 , $35,40,20,20,20$ (G) per group. 

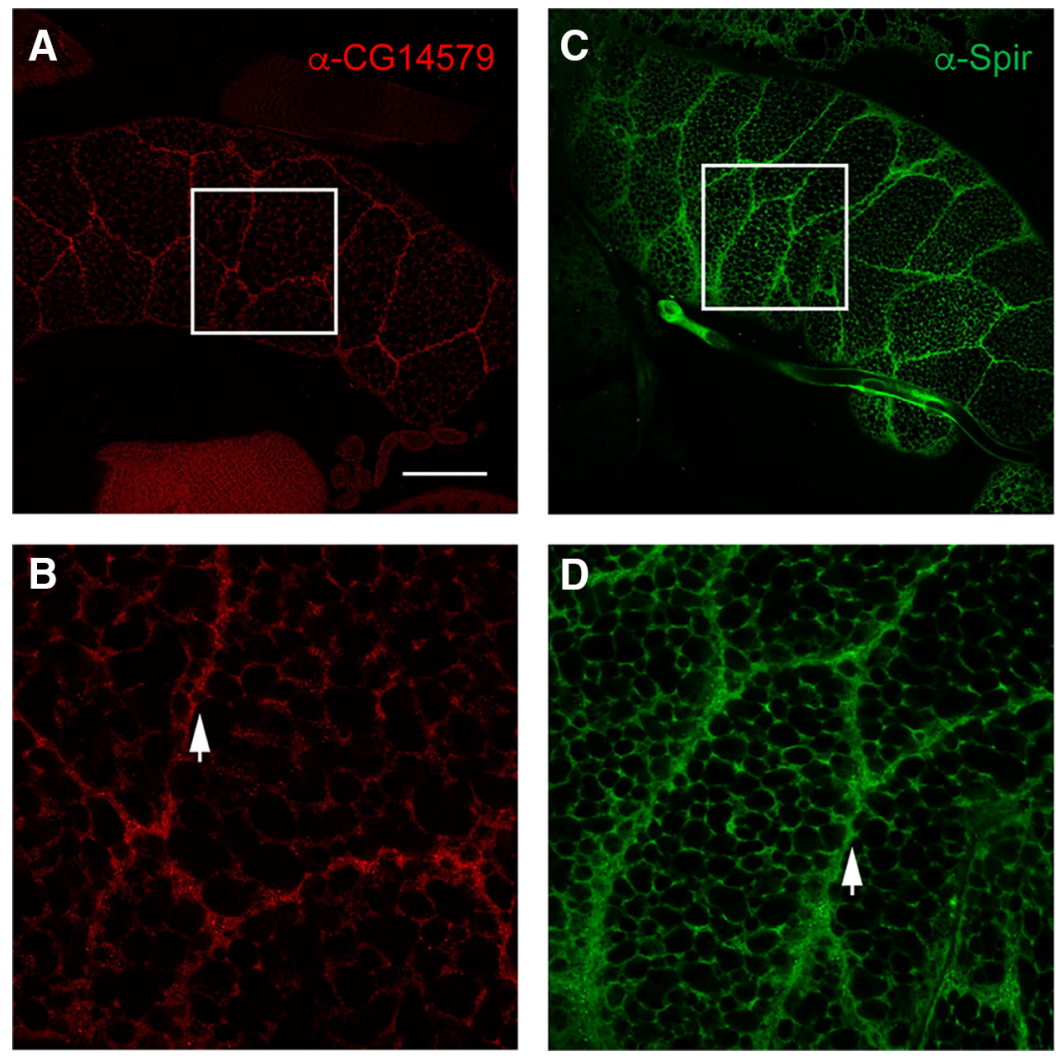

Figure 10. Cellular localization of SPIRE and CCB. A-D, Salivary glands from third instar larvae. A, Monoclonal antibody showing CCB distribution in larval salivary glands. $\boldsymbol{B}$, High-magnification images reveal a punctate pattern in the cytoplasm of the cells from the region marked in $\boldsymbol{A}$. $\boldsymbol{C}$, Monoclonal antibody showing SPIRE distribution in larval salivary glands. $\boldsymbol{D}$, High-magnification images show a similar distribution of SPIRE in the cytoplasm of these cells. Scale bars: $A, C, 65 \mu \mathrm{m} ; \boldsymbol{B}, \boldsymbol{D}, 18 \mu \mathrm{m}$.

A work together to regulate lamination of the various axonal territories within this region (Xie et al., 2017). On the other hand, spontaneous activity is required for proper axonal growth, transcription of signaling molecules and synaptogenesis (Hanson and Landmesser, 2004; Mire et al., 2012; Kirkby et al., 2013; Akin et al., 2019); it is, then, reasonable to conclude that delivery to the growth cone of certain synaptic components through a CCB-dependent mechanism might be required for the growing axons to elicit this spontaneous activity.

The trafficking system investigated here seems to be cargospecific, as it selectively transports specific synaptic proteins such as Synaptobrevin and FasII, but not Syntaxin (Figs. 4-6). This feature suggests the presence of multiple traffic systems to transport synaptic components, which might be functionally related. In this line, there are differential components that mediate high- versus lowfrequency synaptic neurotransmission (Knapek et al., 2010, 2011); if the different components are transported by selective systems, at least two trafficking systems should coexist to complement the transport of synaptic components supporting each type of neurotransmission. This is consistent with the fact that Rab11 belongs to a domain of slow-recycling endosomes.

It seems likely that this CCB transport system could also play important roles in the plasticity of functionally established synapses. It is known that Rab11 is located in mature synaptic vesicles and its overexpression impairs $\mathrm{Ca}^{2+}$-induced exocytosis (Khvotchev et al., 2003). Additionally, neuronal activity-dependent Rab11-driven insertion of AMPA receptors from recycling endosomes and into the postsynaptic plasma membrane underlie the phenomenon of LTP (Park et al., 2004), whereas Rab5 drives the removal of AMPA receptors from synapses into endosomes and contributes to LTD (Brown et al., 2005). Based on the interaction between Rab11 and CCB, we raise the hypothesis that the CCB transport system might perform a similar regulation on the trafficking of synaptic proteins required for synaptic plasticity and memory formation. Although cautious, because of the phylogenetic distance between rodent and fly models, our data provide evidence that CCB is part of the Rab11 vesicular transport system because (1) both proteins colocalize (Fig. $7 I, L)$, (2) both coimmunoprecipitate (Fig. 7P,Q), (3) Rab11 translocation along the axons correlates with CCB levels (Fig. 5), and (4) epistasis studies revealed that Rab11 is positioned downstream of CCB (Fig. 8). Thus, we propose that this delivery system could selectively transport synaptic components involved in specific types of neurotransmission and/or forms of memory. Further investigation of the cargos of this system, especially those already associated to Rab11 (Pavlos and Jahn, 2011; Pylypenko et al., 2016), will test this hypothesis.

Interestingly, Rab11 GTPases are linked to the molecular machinery of the cell through the Rab11-family of interacting proteins (FIPs). For instance, FIP2 specifically interacts with the Actin-based Myosin $\mathrm{Vb}$ motor protein (Hales et al., 2001, 2002; Volpicelli et al., 2002) and this interaction regulates plasma membrane recycling and trafficking of receptors (Pylypenko et al., 2016). Myosin $\mathrm{Vb}$ mobilizes recycling endosomes and AMPA receptors for postsynaptic plasticity (Wang et al., 2008). In general, these FIPs confer specificity in the trafficking of cargos by Rab proteins, and CCB could provide this function. Additionally, SPIRE, an Actin nucleator, shows a very similar subcellular localization to that of $\mathrm{CCB}$ (Fig. 10), and mimics the phenotypes of $c c b$ in axonal transport (Fig. $9 A-F)$. SPIRE belongs to the third family of actin nucleation factors together with the Arp2/3 complex and the Formin family (Quinlan et al., 2005) and is involved in axonal growth, through actin polymerization, and vesicular transport (Kerkhoff et al., 2001). Actindependent long-range transport of vesicles has been previously reported to be mediated by SPIRE and Rab11 (Schuh, 2011). These results underscore the significance of understanding the molecular mechanisms of axonal transport via the actin cytoskeleton, as this transport system plays key roles in cognition and in the deficits associated with neurodegenerative diseases such as Alzheimer's disease (Kommaddi et al., 2018; Martín-Peña et al., 2018). Our results shed light on a newly characterized protein trafficking system which might contribute to the heterogeneity of synapse composition and, thus, on the extended repertoire of functional diversity.

\section{References}

Akin O, Bajar BT, Keles MF, Frye MA, Zipursky SL (2019) Cell-type-specific patterned stimulus-independent neuronal activity in the Drosophila visual system during synapse formation. Neuron 101:894-904.e5.

Brown TC, Tran IC, Backos DS, Esteban JA (2005) NMDA receptordependent activation of the small GTPase Rab5 drives the removal of synaptic AMPA receptors during hippocampal LTD. Neuron 45:81-94. 
Chia PH, Chen B, Li P, Rosen MK, Shen K (2014) Local F-actin network links synapse formation and axon branching. Cell 156:208-220.

Dietzl G, Chen D, Schnorrer F, Su KC, Barinova Y, Fellner M, Gasser B, Kinsey K, Oppel S, Scheiblauer S, Couto A, Marra V, Keleman K, Dickson BJ (2007) A genome-wide transgenic RNAi library for conditional gene inactivation in Drosophila. Nature 448:151-156.

Fushima K, Tsujimura H (2007) Precise control of fasciclin II expression is required for adult mushroom body development in Drosophila. Dev Growth Differ 49:215-227.

Georgiou M, Tear G (2003) The N-terminal and transmembrane domains of commissureless are necessary for its function and trafficking within neurons. Mech Dev 120:1009-1019.

Giot L,Bader JS, Brouwer C, Chaudhuri A, Kuang B, Li Y, Hao YL, Ooi CE, Godwin B, Vitols E, Vijayadamodar G, Pochart P, Machineni H, Welsh M, Kong Y, Zerhusen B, Malcolm R, Varrone Z, Collis A, Minto M, et al. (2003) A protein interaction map of Drosophila melanogaster. Science 302:1727-1736.

Grosshans BL, Ortiz D, Novick P (2006) Rabs and their effectors: achieving specificity in membrane traffic. Proc Natl Acad Sci U SA 103: 11821-11827.

Hales CM, Griner R, Hobdy-Henderson KC, Dorn MC, Hardy D, Kumar R, Navarre J, Chan EK, Lapierre LA, Goldenring JR (2001) Identification and characterization of a family of Rab11-interacting proteins. J Biol Chem 276:39067-39075.

Hales CM, Vaerman JP, Goldenring JR (2002) Rab11 family interacting protein 2 associates with myosin $\mathrm{vb}$ and regulates plasma membrane recycling. J Biol Chem 277:50415-50421.

Hanson MG, Landmesser LT (2004) Normal patterns of spontaneous activity are required for correct motor axon guidance and the expression of specific guidance molecules. Neuron 43:687-701.

Heisenberg M, Borst A, Wagner S, Byers D (1985) Drosophila mushroom body mutants are deficient in olfactory learning. J Neurogenet 2:1-30.

Hirokawa N, Niwa S, Tanaka Y (2010) Molecular motors in neurons: transport mechanisms and roles in brain function, development, and disease. Neuron 68:610-638.

Kalidas S, Smith DP (2002) Novel genomic cDNA hybrids produce effective RNA interference in adult Drosophila. Neuron 33:177-184.

Kerkhoff E, Simpson JC, Leberfinger CB, Otto IM, Doerks T, Bork P, Rapp UR, Raabe T, Pepperkok R (2001) The spir actin organizers are involved in vesicle transport processes. Curr Biol 11:1963-1968.

Khodosh R, Augsburger A, Schwarz TL, Garrity PA (2006) Bchs, a BEACH domain protein, antagonizes Rab11 in synapse morphogenesis and other developmental events. Development 133:4655-4665.

Khvotchev MV, Ren M, Takamori S, Jahn R, Südhof TC (2003) Divergent functions of neuronal Rab1 $1 \mathrm{~b}$ in $\mathrm{Ca}^{2+}$-regulated versus constitutive exocytosis. J Neurosci 23:10531-10539.

King SM (2018) Turning dyneins off bends cilia. Cytoskeleton 75:372-381.

Kirkby LA, Sack GS, Firl A, Feller MB (2013) A role for correlated spontaneous activity in the assembly of neural circuits. Neuron 80:1129-1144.

Knapek S, Gerber B, Tanimoto H (2010) Synapsin is selectively required for anesthesia-sensitive memory. Learn Mem 17:76-79.

Knapek S, Sigrist S, Tanimoto H (2011) Bruchpilot, a synaptic active zone protein for anesthesia-resistant memory. J Neurosci 31:3453-3458.

Kommaddi RP, Das D, Karunakaran S, Nanguneri S, Bapat D, Ray A, Shaw E, Bennett DA, Nair D, Ravindranath V (2018) A $\beta$ mediates F-actin disassembly in dendritic spines leading to cognitive deficits in Alzheimer's disease. J Neurosci 38:1085-1099.

Kurusu M, Awasaki T, Masuda-Nakagawa LM, Kawauchi H, Ito K, Furukubo-Tokunaga K (2002) Embryonic and larval development of the Drosophila mushroom bodies: concentric layer subdivisions and the role of fasciclin II. Development 129:409-419.

Martín-Peña A, Acebes A, Rodríguez JR, Sorribes A, de Polavieja GG, Fernández-Fúnez P, Ferrús A (2006) Age-independent synaptogenesis by phosphoinositide 3 kinase. J Neurosci 26:10199-10208.

Martín-Peña A, Acebes A, Rodríguez JR, Chevalier V, Casas-Tinto S, Triphan T, Strauss R, Ferrús A (2014) Cell types and coincident synapses in the ellipsoid body of Drosophila. Eur J Neurosci 39:1586-1601.

Martín-Peña A, Rincón-Limas DE, Fernandez-Fúnez P (2018) Engineered
Hsp70 chaperones prevent A $\beta 42$-induced memory impairments in a Drosophila model of Alzheimer's disease. Sci Rep 8:9915.

Meyer MP, Smith SJ (2006) Evidence from in vivo imaging that synaptogenesis guides the growth and branching of axonal arbors by two distinct mechanisms. J Neurosci 26:3604-3614.

Mire E, Mezzera C, Leyva-Díaz E, Paternain AV, Squarzoni P, Bluy L, Castillo-Paterna M, López MJ, Peregrín S, Tessier-Lavigne M, Garel S, Galcerán J, Lerma J, López-Bendito G (2012) Spontaneous activity regulates Robol transcription to mediate a switch in thalamocortical axon growth. Nat Neurosci 15:1134-1143.

Murthy M, Garza D, Scheller RH, Schwarz TL (2003) Mutations in the exocyst component Sec5 disrupt neuronal membrane traffic, but neurotransmitter release persists. Neuron 37:433-447.

O'Kane CJ, Gehring WJ (1987) Detection in situ of genomic regulatory elements in Drosophila. Proc Natl Acad Sci U S A 84:9123-9127.

Park M, Penick EC, Edwards JG, Kauer JA, Ehlers MD (2004) Recycling endosomes supply AMPA receptors for LTP. Science 305:1972-1975.

Pavlos NJ, Jahn R (2011) Distinct yet overlapping roles of rab GTPases on synaptic vesicles. Small GTPases 2:77-81.

Pylypenko O, Welz T, Tittel J, Kollmar M, Chardon F, Malherbe G, Weiss S, Michel CI, Samol-Wolf A, Grasskamp AT, Hume A, Goud B, Baron B, England P, Titus MA, Schwille P, Weidemann T, Houdusse A, Kerkhoff E (2016) Coordinated recruitment of Spir actin nucleators and myosin V motors to Rab11 vesicle membranes. eLife 5:e17523.

Quinlan ME, Heuser JE, Kerkhoff E, Mullins RD (2005) Drosophila spire is an actin nucleation factor. Nature 433:382-388.

Renn SC, Armstrong JD, Yang M, Wang Z, An X, Kaiser K, Taghert PH (1999) Genetic analysis of the Drosophila ellipsoid body neuropil: organization and development of the central complex. J Neurobiol 41: 189-207.

Romero-Pozuelo J, Dason JS, Atwood HL, Ferrús A (2007) Chronic and acute alterations in the functional levels of frequenins 1 and 2 reveal their roles in synaptic transmission and axon terminal morphology. Eur J Neurosci 26:2428-2443.

Rottner K, Faix J, Bogdan S, Linder S, Kerkhoff E (2017) Actin assembly mechanisms at a glance. J Cell Sci 130:3427-3435.

Schuh M (2011) An actin-dependent mechanism for long-range vesicle transport. Nat Cell Biol 13:1431-1436.

Sönnichsen B, De Renzis S, Nielsen E, Rietdorf J, Zerial M (2000) Distinct membrane domains on endosomes in the recycling pathway visualized by multicolor imaging of Rab4, Rab5, and Rab11. J Cell Biol 149:901-914.

Strauss R, Heisenberg M (1993) A higher control center of locomotor behavior in the Drosophila brain. J Neurosci 13:1852-1861.

Tear G, Harris R, Sutaria S, Kilomanski K, Goodman CS, Seeger MA (1996) commissureless controls growth cone guidance across the CNS midline in Drosophila and encodes a novel membrane protein. Neuron 16:501-514.

Tittel J, Welz T, Czogalla A, Dietrich S, Samol-Wolf A, Schulte M, Schwille P, Weidemann T, Kerkhoff E (2015) Membrane targeting of the Spirformin actin nucleator complex requires a sequential handshake of polar interactions. J Biol Chem 290:6428-6444.

Volpicelli LA, Lah JJ, Fang G, Goldenring JR, Levey AI (2002) Rab1la and myosin $\mathrm{Vb}$ regulate recycling of the $\mathrm{M}_{4}$ muscarinic acetylcholine receptor. J Neurosci 22:9776-9784.

Wang Z, Edwards JG, Riley N, Provance DW Jr, Karcher R, Li XD, Davison IG, Ikebe M, Mercer JA, Kauer JA, Ehlers MD (2008) Myosin vb mobilizes recycling endosomes and AMPA receptors for postsynaptic plasticity. Cell 135:535-548.

Winther AM, Siviter RJ, Isaac RE, Predel R, Nässel DR (2003) Neuronal expression of tachykinin-related peptides and gene transcript during postembryonic development of Drosophila. J Comp Neurol 464:180-196.

Xie X, Tabuchi M, Brown MP, Mitchell SP, Wu MN, Kolodkin AL (2017) The laminar organization of the Drosophila ellipsoid body is semaphorindependent and prevents the formation of ectopic synaptic connections. eLife 6:e25328.

Yu HH, Huang AS, Kolodkin AL (2000) Semaphorin-1a acts in concert with the cell adhesion molecules fasciclin II and connectin to regulate axon fasciculation in Drosophila. Genetics 156:723-731. 